\title{
Systematic dissection of a complex gut bacterial community
}

${ }^{1}$ Department of Gastroenterology, Stanford University School of Medicine, Stanford, CA 94305, USA

${ }^{2}$ Department of Bioengineering, Stanford University, Stanford, CA 94305, USA

${ }^{3}$ Chan Zuckerberg Biohub, San Francisco, CA 94158, USA

${ }^{4}$ ChEM-H Institute, Stanford University, Stanford, CA 94305, USA

${ }^{5}$ Environmental Genomics and Systems Biology Division, Lawrence Berkeley National Laboratory,

13 Berkeley, CA 94720, USA

${ }^{6}$ Department of Plant and Microbial Biology, University of California, Berkeley, CA 94720, USA

${ }^{7}$ Department of Microbiology and Immunology, Stanford University School of Medicine, Stanford

University, Stanford, CA 94305, USA

${ }^{*}$ Equal contribution

†Correspondence: kchuang@stanford.edu, fischbach@fischbachgroup.org

Lead author: Michael Fischbach (fischbach@fischbachgroup.org) 


\section{ABSTRACT}

Efforts to model the gut microbiome have yielded important insights into the mechanisms of interspecies interactions, the impact of priority effects on ecosystem dynamics, and the role of diet and nutrient availability in determining community composition. However, the model communities studied to date have been defined or complex but not both, limiting their utility. Here, we construct a defined community of 104 bacterial strains composed of the most common taxa from the human gut microbiota. By propagating this community in growth media missing one amino acid at a time, we show that branchedchain amino acids have an outsize impact on community structure and identify a pathway in Clostridium sporogenes for generating ATP from arginine. We constructed and propagated the complete set of singlestrain dropout communities, revealing a sparse network of strain-strain interactions including a novel interaction between $C$. sporogenes and Lactococcus lactis driven by metabolism. This work forms a 32 foundation for studying strain-strain and strain-nutrient interactions in highly complex defined communities, and it provides a starting point for interrogating the rules of synthetic ecology at the $100+$ strain scale. 


\section{INTRODUCTION}

Model systems have proven invaluable for the development of mechanistic insight in biology (Müller and Grossniklaus, 2010). Although much has been learned from detailed studies of individual gut commensal species (Cullen et al., 2015; Cuskin et al., 2015; Sonnenburg et al., 2010; Wexler and Goodman, 2017), models of the gut microbiome are less well developed (Blasche et al., 2017; Pacheco and Segrè, 2019; Walter et al., 2018; Widder et al., 2016; Xavier, 2011). Pioneering efforts showed that a synthetic community can model the impact of diet on the microbiome (Faith et al., 2011), identified genes required for Bacteroides thetaiotaomicron growth in the mouse intestine in the presence of a 15-member community (Goodman et al., 2009), and demonstrated that complex communities composed of species isolated from a single donor can stably colonize mice (Goodman et al., 2011). More recently, defined communities of up to 20 naturally occurring (Abreu et al., 2019; Friedman et al., 2017; Gutiérrez and Garrido, 2019; Hoek et al., 2016; Medlock et al., 2018; Patnode et al., 2019; Sanchez-Gorostiaga et al., 2019; Yurtsev et al., 2016) or genetically engineered (Hart et al., 2019; Hsu et al., 2019; Kong et al., 2018; Mee et al., 2014; Ziesack et al., 2019) bacterial strains have been studied in vitro or in mice, revealing insights into the mechanisms of interspecies interactions. Complex but undefined communities from the gut, soil, and plants have also been studied in detail, illuminating the role of priority effects and the environment-especially nutrient availability-in determining community composition and dynamics (Aranda-Díaz et al., 2020; Goldford et al., 2018; Martínez et al., 2018).

Although these studies have provided foundational insights into the ecology of the gut microbiota, the synthetic communities used have been defined or complex, but not both. An optimal model system would have both features: Near-native complexity would allow a model microbiome to capture properties of an ecosystem that are missing from simpler model systems, including emergent phenomena such as resilience to perturbation (Dethlefsen and Relman, 2011; $\mathrm{Ng}$ et al., 2019) and cooperative metabolism (Morris et al., 2013). Moreover, complex consortia are a promising starting point for in vivo studies of the gut microbiome, for which they are better suited to model community-level phenomena such as immune modulation and the formation of structured multispecies biofilms.

Complete definition (i.e., communities composed entirely of known organisms) would enable reductionist experiments to probe mechanism. Studies with relatively simple defined communities have demonstrated the power of strain dropout and gene deletion experiments for probing community function. The ability to construct communities with defined composition is especially relevant in the context of experiments testing whether phenotypes can be transferred to germ-free mice via fecal transplant (Gopalakrishnan et al., 2018; Ridaura et al., 2013; Routy et al., 2018). At present, since transplanted communities are typically undefined, it is difficult to uncover the mechanisms underlying these phenomena. A defined model system of sufficient complexity would enable reductionist follow-up experiments, bringing the gut microbiome in line with other model systems in which mechanistic studies are possible. 
Here, echoing efforts focused on the plant microbiota (Bai et al., 2015; Carlström et al., 2019; Lebeis et al., 2015), we constructed a complex defined community that contains the most prevalent bacterial species in the human gut microbiome. We demonstrate that the assembly of this 104-member community is reproducible even for very low abundance species. By systematically perturbing this community and its growth medium, we uncover a set of strain-nutrient and strain-strain (e.g. syntrophic) interactions that underlie its composition. This work constitutes a starting point for studying complex synthetic communities at high resolution across orders of magnitude of relative abundance.

\section{RESULTS}

\section{Designing and building a complex synthetic community}

We set out to design a community consisting of the most common bacterial strains in the human gut microbiome. We analyzed metagenomic sequence data from the NIH Human Microbiome Project (HMP) to determine the most prevalent organisms - those that were present in the largest proportion of subjects, regardless of abundance. Although the HMP is not broadly representative of microbiomes from diverse geographies and ethnicities (Deschasaux et al., 2018; He et al., 2018; Sonnenburg and Sonnenburg, 2019), this data set was well suited to our purposes since it was sequenced at very high depth, enabling us to identify low-abundance organisms that are nevertheless highly prevalent (Kraal et al., 2014). After rank-ordering bacterial strains by prevalence, we found that $\sim 20 \%$ (166/844) were present in $>45 \%$ of the HMP subjects. Of these 166 strains, we were able to obtain 99 from culture collections or individual laboratories (Figure 1A). The profiled strains of three additional species were unavailable, so we used alternative strains of the same species (Lactococcus lactis subsp. lactis II1403, Bacteroides xylanisolvens DSM 18836, and Megasphaera sp. DSM 102144). We added two additional strains to enable downstream experiments: Ruminococcus bromii ATCC 27255, a keystone species in polysaccharide utilization (Ze et al., 2012); and Clostridium sporogenes ATCC 15579, a model gut Clostridium species for which genetic tools are available (Dodd et al., 2017; Funabashi et al., 2020; Guo et al., 2019). Together, these 104 strains resemble the phylogenetic distribution of a typical Western human gut community (Figure S1). Notably, unlike other defined communities used to model the gut microbiome, our consortium is within $\sim 2$-fold of the estimated number of strains in a typical human gut (Faith et al., 2013; Qin et al., 2010).

A streamlined strain growth protocol simplified the assembly of the complete community and singlestrain dropouts. By testing the growth of each strain in a panel of candidate growth media, we identified two media, at least one of which supports the growth of all 104 strains (Table S1). Each culture was passaged daily 2-3 times with dilution into fresh medium. Growth rates, carrying capacities, and time of entry into stationary phase varied widely across strains and media; by passaging fast-growing strains more frequently than slow-growing organisms, we synchronized culture saturation to the extent possible. Before 
mixing individually cultured strains, we adjusted the volumes of each culture to achieve similar optical densities. We confirmed that these cultures were pure using metagenomic sequencing and high accuracy read mapping, as described in the next section.

\section{Development of a highly accurate metagenomic read-mapping pipeline}

Having assembled a community of 104 strains, we next addressed how to quantify the abundance of each strain accurately, a major challenge in light of our expectation that some strains would be present at low abundance. Various strains in the community have identical $16 \mathrm{~S}$ hypervariable sequences, ruling out $16 \mathrm{~S}$ amplicon-based methods. We considered designing a custom amplicon-based pipeline, but such an approach would require the design and validation of new primer sets for future communities, which we wished to avoid. Instead, we sought to use metagenomic sequencing as a means of quantifying community composition.

To test the performance of existing metagenomic analysis tools, we generated three 'ground truth' data sets. The first two consisted of simulated reads generated from the assembled genome sequences of each strain: one in which all 104 strains were equally abundant (to test sensitivity and specificity), and another in which strain abundance varied over five orders of magnitude (to test dynamic range). The third set consisted of actual reads derived from sequencing each strain individually using the same protocol on the same sequencing instrument used for subsequent community analyses. This data set allowed us to account for biases introduced by library construction and sequencing.

We found that metagenomic read mappers based on a combination of Bowtie2 (Langmead and Salzberg, 2012) and SAMtools (Li et al., 2009) were sensitive but inaccurate: there was substantial mismapping of reads from one strain to others, such that whole-genome sequencing data from an individual strain was often interpreted as having arisen from multiple strains. Read mis-mapping from any abundant strain would therefore create noise that exceeds signal from low-abundance strains, degrading accuracy. In contrast, algorithms that focus on a few universal genes or unique k-mers such as MetaPhlan2 (Truong et al., 2015), MIDAS (Nayfach et al., 2016), Kraken2/Bracken (Lu et al., 2017; Wood et al., 2019), IGGsearch (Nayfach et al., 2019), or Sourmash (Titus Brown and Irber, 2016) were generally accurate to the species level, but since they only use a small fraction of the reads $(<1 \%)$, their ability to detect lowabundance or closely related strains is limited.

To address these challenges, we developed a new algorithm, NinjaMap (Figure 2A). Taking advantage of the fact that every strain in our community has been sequenced, NinjaMap quantifies strain abundances with high accuracy across $>6$ orders of magnitude. In brief, NinjaMap considers every read from a sample. If a read does not match perfectly to any of the genomes in the community (typically 3-4\% of the reads), it is tabulated but not assigned. If a read has a perfect match to only one strain, it is assigned unambiguously to that strain. If a read matches more than one strain perfectly, it is temporarily placed in 
escrow. After all of the unambiguous assignments are made, an initial estimate of the relative abundance of each strain is computed. Reads in escrow are then fractionally assigned in proportion to the relative abundance of each strain, normalized by the total size of the genomic regions available for unique mapping to avoid bias in favor of strains with large or phylogenetically distinct genome sequences. Finally, relative abundances are computed.

To assess the performance of NinjaMap, we performed two tests. First, we assessed the degree of read mis-mapping from and into each strain's ledger-e.g., we quantified how many reads from Bacteroides ovatus ATCC 8483 were mis-assigned to other strains (which would underestimate its abundance in a community), and how many reads from other strains were mis-assigned to $B$. ovatus (which would overestimate its abundance). For simulated reads, most instances of read mis-mapping resulted in relative abundance errors $<10^{-4}$ (Figure S2A). For actual reads, mis-mapping was more frequent but still typically below a threshold of $10^{-3}$; most mis-mapping arose from deviations between the database genome sequence and the actual sequence of the strain in our collection (Figure S2B). In general, if the abundance of a strain in a community was within 10-fold of what would be expected from mis-mapping, we excluded the strain from analyses (Methods).

Second, we used Ninjamap to analyze simulated reads from a 104-strain community. We found that this tool can accurately quantify strains with abundances as low as $10^{-5}$ in the context of a mixed community of known composition (Figure 2B). Thus, NinjaMap is capable of quantifying strains accurately over a wide dynamic range of relative abundances.

\section{Community construction is highly reproducible}

Our protocol for assembling communities with >100 members involves several growth passages and liquid transfer steps, and we were concerned that variability in any step of our protocol could make it difficult to interpret results. To address this concern, we measured the degree of reproducibility in community composition data by constructing and propagating the 104-member community multiple times. We included technical replicates to assess variation in bacterial growth, DNA extraction, and sequencing, and biological replicates to determine the impact of differences in the preparation of the inocula. We propagated the communities for $48 \mathrm{~h}$ and extracted DNA for sequencing at $0,12,24$, and $48 \mathrm{~h}$.

Despite our attempts to inoculate equal densities of each strain, the range of densities at $t=0$ spanned several orders of magnitude (Figure 2B), with a mean $\log _{10}$ (relative abundance) of $-2.5 \pm 0.8$ for all detectable strains. Nonetheless, 95/104 strains were detectable at $t=0$; the remaining strains were below the limit of detection or had abundances that could potentially be explained by read mis-mapping. The communities reached a relatively stable configuration by $12 \mathrm{~h}$ (Figure 2B), with a remarkable degree of reproducibility among biological replicates (Figure 2C). Technical replicates were even more similar 173 (Figure 2D), indicating that community growth, DNA extraction, and sequencing contributed only modestly 
174 to variability. Notably, very low-abundance strains $\left(<10^{-4}\right)$ were only slightly more variable than high175 abundance strains (Figure 2C). Taken together, these results indicate that community composition is 176 robust to experimental variation.

\section{A nutrient drop-out screen to map strain-nutrient interactions in the community}

We next sought to explore the network of strain-nutrient interactions in the community. Although much is known about polysaccharide foraging by gut commensals (Martens et al., 2014), far less is known about amino acid utilization, so we performed the experiment in a defined growth medium (SAAC) from which we could remove one amino acid at a time. Since amino acids are often utilized in pairs (Nisman, 1954; Smith and Macfarlane, 1997), eliminating one at a time from a complete background rather than adding one at a time to a null background has a greater potential to reveal phenotypes relevant to community function. Moreover, performing this screen in the context of a complex community (as opposed to the traditional practice of analyzing the growth of isolated strains) enables us to study communitydependent effects such as nutrient competition or mutualism-dependent nutrient utilization.

To map strain-amino acid interactions, we constructed the 104-member community by mixing cultures of each strain propagated in a rich growth medium. We then sub-cultured this consortium in 20 defined growth media, each deficient in a single amino acid; the complete defined medium was used as a control (Figure 3A). Samples were taken at $48 \mathrm{~h}$ and metagenomic sequencing data were analyzed to determine the impact of amino acid deficiency on the relative abundance of each strain.

\section{Global analysis of strain-amino acid interactions}

To identify strain-amino acid interactions, we tabulated strains whose relative abundance deviated significantly from the mean across conditions, taking advantage of the fact that that most amino acid dropouts had little effect on most strains (Figure 3B, Methods). When the community was propagated in the complete defined medium, relative abundances spanned $>6$ orders of magnitude. $36 \%$ of the strains were present at $10^{-4}-10^{-2}$ relative abundance, 8 strains were $>10^{-2}$ and 50 were $<10^{-4}$ (Figure 3B). NinjaMap was sensitive to strains with relative abundances as low as $10^{-6}$, enabling us to quantify the $56 \%$ of strains that were below the $10^{-3}$ limit of detection commonly used for metagenomic analyses (Franzosa et al., 2015). Our system is therefore capable of studying low-abundance microbes, some of which are known to have a large biological impact (Buffie et al., 2015; Funabashi et al., 2020).

To identify significant responses, we calculated the standard deviation of the relative abundance of 206 each strain across experiments and computed z-scores (Figure 3C). Strain-amino acid interactions that were previously identified in mono-culture studies were also observed in our community format. Anaerostipes caccae, whose growth is stimulated by methionine (Soto-Martin et al., 2020), decreased in relative abundance in a community grown in methionine-deficient medium ( $z=-3.48)$. Likewise, $C$. 
sporogenes was impeded by the absence of leucine ( $z=-2.56)$, a substrate it oxidatively decarboxylates to isovalerate to generate electrons (Guo et al., 2019). These observations demonstrate that even though $>100$ strains are competing for the same nutrients, the effects of eliminating one amino acid on the growth of one strain are readily observable in the context of a complex community.

Most strains responded to the removal of $\leq 4$ amino acids. Moreover, relative abundances showed little variability, with a mean standard deviation of $\log _{10}$ (relative abundance) across strains $<0.43$. Only three strains, all of which are Firmicutes, were responsive to more amino acids: Lactococcus lactis DSM 20729, Clostridium sporogenes ATCC 15579, and Lactobacillus ruminis ATCC 25644 (Figure S3, Table S2). Thus, under these growth conditions, most strains are largely insensitive to amino acid removal while a small minority are highly responsive. We note that the response of a strain to amino acid removal may be direct (e.g. due to utilization for energy) or indirect (e.g. amino acid removal impacts an interacting strain).

In contrast, amino acids varied widely in terms of their impact on community composition (Figure 3D). More than half of the strains responded to cysteine removal, likely due to its effect as a reducing agent. More than $5 \%$ of the strains responded to methionine, histidine, isoleucine, arginine, valine, and tyrosine, while for eight amino acids there were no significant changes to the community at all (Figure 3D). Interestingly, there were large differences among similar amino acids: no strains responded to lysine, while $10.6 \%$ and $7.6 \%$ of the strains responded to histidine and arginine, respectively. The removal of isoleucine, leucine, and arginine had a particularly large impact on community structure: $C$. sporogenes and $L$. lactis, the two most abundant strains when grown in complete defined medium, each decreased $>500$-fold in relative abundance when any of these amino acids were removed. Thus, certain amino acids are 'keystone' nutrients that play an important role in determining community composition.

\section{C. sporogenes uses arginine to generate ATP}

Among the 86 candidate strain-amino acid interactions revealed by our screen, we were particularly intrigued by those involving $C$. sporogenes. Although $C$. sporogenes can oxidize and reduce aromatic amino acids (Dodd et al., 2017), its relative abundance was unaffected by the removal of phenylalanine, tyrosine, or tryptophan (Figure S4). In contrast, the removal of leucine, isoleucine, and arginine each had large impact on its fitness in the community. The second strongest phenotype was a decrease in relative abundance in the absence of arginine (Figure 3E); although $C$. sporogenes is known to metabolize arginine (Venugopal and Nadkarni, 1977; Wildenauer and Winter, 1986), no impact of arginine on growth or energy metabolism had been observed in prior work. To validate and characterize this interaction, we compared $C$. sporogenes growth in complete defined versus arginine-deficient medium. Although $C$. 242 sporogenes grew well in complete defined medium, it exhibited a large growth defect in the absence of 243 arginine (Figure 3F), indicating that this amino acid is an important substrate for growth. 
C. sporogenes can use other amino acids as substrates to support ATP synthesis (Dodd et al., 2017). Hypothesizing that the same is true for arginine, we incubated wild-type C. sporogenes in a culture medium deficient in substrates for ATP synthesis. Upon addition of arginine, intracellular ATP levels rose sharply (Figure 3G), indicating that C. sporogenes generates ATP (directly or indirectly) from arginine.

To identify the enzymes involved in this process, we parsed the $C$. sporogenes genome for pathways known to capture energy from arginine. This search yielded candidate genes for each of the three steps in the arginine deiminase pathway (Figure $3 \mathbf{H}$ ), which catalyzes the net conversion of arginine to ornithine plus $\mathrm{CO}_{2}$ and two equivalents of ammonium, generating one equivalent of ATP (Cunin et al., 1986). Using a method we recently developed to construct scarless deletions in C. sporogenes (Guo et al., 2019), we generated strains deficient in the putative arginine deiminase (CLOSPO_00894, $\Delta$ adi) or ornithine carbamoyltransferase (CLOSPO_02415, _otc). The $\Delta$ otc mutant was unable to generate ATP in response to arginine provision, consistent with a role for the arginine deiminase pathway in $C$. sporogenes energy production (Figure 3G). In contrast, the $\Delta$ adi mutant showed no defect in arginine-induced ATP production (Figure S5A), suggesting the possibility of an alternative pathway to generate citrulline from arginine. Consistent with these observations, the $\Delta$ otc mutant (but not the $\Delta$ adi mutant) was deficient in growth in complete defined medium (Figures 3F, Figure S5B). The deficiency was partial, suggesting that an alternative pathway can generate energy from arginine under these conditions. Together, these results show that arginine metabolism by the arginine deiminase pathway contributes directly to the cellular ATP pool, augmenting our understanding of how amino acid metabolic pathways contribute to the fitness of a prominent gut commensal within a complex community.

\section{A strain drop-out screen to map strain-strain interactions}

Next, we sought to map strain-strain interactions within the community. A wide variety of interaction types have been characterized (Little et al., 2008; Shank and Kolter, 2009), including mutualistic or commensal interactions based on nutrient exchange (e.g. syntrophies and secondary fermentation) (Morris in a complex community (Bairey et al., 2016).

To address this gap in knowledge, we constructed all 104 single-strain dropout communities (Figure 4A, Methods). These 103-member communities as well as the full community were grown in complete defined medium, and samples were taken at 0 and $48 \mathrm{~h}$ to assay growth dynamics over the 
We observed that the relative abundances in our data were correlated due to batch effects arising from the order in which communities were constructed (Figure S6); they clustered naturally into four sets. The two smallest sets ( 8 and 12 dropouts) were not considered further due to challenges in evaluating statistical significance. Within the two larger sets-60 and 24 dropouts-the relative abundances of each strain across samples were tightly distributed (Figure 4B, S7), enabling us to identify statistically significant responses using z-scores.

To test whether the single-strain dropout communities were deficient in the intended strain, we 286 compared the relative abundance of each strain across all samples (Figure 4B, Table S3). For 71 of 84 communities, the strain we intended to remove had $z<-3$, indicating that the dropout was successful. In the remaining 13 communities, the intended strain was either below our limit of detection or was detected at $48 \mathrm{~h}$; in both cases, the corresponding communities were not considered further (Methods).

Two additional filters were applied, resulting in the removal of six additional samples. First, five samples that had $<10^{6}$ reads were not considered further due to the underrepresentation of low-abundance strains. Second, the E. siraeum dropout was missing an additional strain, A. caccae, and was removed from further consideration.

\section{Global analysis of strain-strain interactions}

We analyzed the remaining 65 communities (Table S4) to identify strains whose relative abundance changed significantly in response to the absence of another strain. Putative interactions $a \rightarrow b$ (where the arrow indicates that strain $b$ increases or decreases in relative abundance in response to the absence of strain a) were considered further if they had $|z|>2$. We removed potentially spurious interactions that could have resulted from read mis-mapping or relative abundances near the lower limit of detection (Methods).

Despite the community's complexity, large effects could be observed when certain strains were dropped out. For example, removing Acidaminococcus sp. D21 resulted in a 4-fold increase in the abundance of Acidaminococcus fermentans DSM 20731 (Figure 4B), presumably reflecting nutrient competition between strains of the same genus. The relative abundances of $A$. fermentans DSM 20731 were tightly distributed in other samples (Figure 4A, inset), so the change in relative abundance due to Acidaminococcus sp. D21 dropout was highly significant ( $z=5.1)$.

Most of the strain dropouts affected $<5 \%$ of the other strains (Figure 4C). However, seven strainswhen dropped out-impacted $>10 \%$ of the remaining strains positively or negatively. Two of those strains, Acidaminococcus sp. D21 and A. caccae DSM 14662, were present at high relative abundance, so it is not surprising that relative abundances within the community redistribute upon their removal. However, the remaining strains-Dorea formicigenerans ATCC 27755, Dialister invisus DSM 15470, Bacteroides dorei 313 5_1_36/D4, B. fragilis 3_1_12, and C. sporogenes ATCC 15579—were present at a relative abundance of 
314 just $\sim 10^{-3}-10^{-2}$, yet their removal altered the relative abundance of $>7$ other strains (Figure 4D). 315 Conversely, four strains (Bacteroides sp. 2_1_16, Bacteroides cellulosilyticus DSM 14838, Bacteroides 316 ovatus ATCC 8483, and Mitsuokella multacida DSM 20544) had relative abundances $>1 \%$ but affected $<2$ 317 strains when removed (Figure 4D), consistent with functional redundancy or another mechanism by which 318 these strains are insulated from the rest of the community. Thus, the community is largely insensitive to 319 strain removal even though a small subset of strains exhibit keystone-like properties.

\section{Validating candidate interactions in binary culture}

Next, we tested whether interactions uncovered by the strain dropout screen could be observed in binary culture. Interactions can be direct or indirect (i.e. involve one or more additional strains), and contextindependent or dependent (i.e. occur only in the background of a complex community). Only those interactions that are direct and context-independent would be reproducible in binary culture. We focused on interactions with $z<-2$ (rather than $z>2$ ), since the former involve growth promotion and are therefore simpler to validate in binary culture (Table S4).

We selected 32 candidate interactions with highly negative z-scores for further characterization. For these strain pairs, we compared the optical density of each strain when grown in monoculture versus co-culture, using the same defined medium as in the dropout screen. 23/32 strain pairs exhibited an increased carrying capacity relative to an additive growth model (Figure 4E) and/or decreased time to saturation in co-culture (Figure S8). These data indicate that the screen is an effective means of uncovering direct, context-independent strain-strain interactions. For 9/32 strain pairs, no interaction was observed in binary culture. While these negative results could be due to imperfections in the strain dropout experiment or analysis, they might also suggest that certain interactions can only be observed in the setting of a complex community.

We were intrigued that the level of Lactococcus lactis DSM 20729 decreased when C. sporogenes ATCC 15579 was dropped out of the community $(z=-2.10)$. Although the effect size was small, the distribution of $L$. lactis relative abundances was particularly narrow, so the absence of $C$. sporogenes disrupted the growth of an otherwise context-insensitive strain.

To determine whether this interaction occurred in binary co-culture, we cultured C. sporogenes and $L$. lactis individually or together; $C$. sporogenes grew rapidly in defined medium while $L$. lactis was unable to grow on its own. The optical density of the co-culture was substantially higher than the sum of the optical densities of the individual cultures, suggesting that at least one of the strains grew more robustly in coculture (Figure 4F). By counting colonies from the co-culture, we determined that $C$. sporogenes levels were unaffected but the density of $L$. lactis increased $\sim 10$-fold (Figure $4 \mathbf{G}$ ). These results validate the postulated interaction between $C$. sporogenes and L. lactis and suggest the possibility that the apparent 
349 abundance of $C$. sporogenes. More broadly, our findings show that a growth stimulatory interaction 350 between two strains can manifest even over one round of growth in the presence of 102 other strains.

\section{DISCUSSION}

Our experimental system has three important features. First, by developing a community that is both defined and reasonably complex, we have generated a model system that will likely capture much of the biology of a native microbiome. Future refinements are needed, including additional bacterial strains to occupy unfilled niches as well as archaea, fungi, and viruses, all of which are important components of the native ecosystem.

Second, the computational pipeline we developed for read mapping makes it possible to analyze complex defined communities with high precision. Community structure can be quantified across six orders of magnitude in relative abundance, enabling the interrogation of low-abundance community members that play an important role in community function and dynamics (Buffie et al., 2015; Funabashi et al., 2020). The degree of technical and biological reproducibility (Figure 2E) is remarkable in a system this complex, which bodes well for future experimental efforts.

Third, the nutrient and strain dropout assays are a powerful format for probing the interactions that underlie community dynamics. The amino acid dropout screen tested 2,080 potential interactions (104 strains $\times 20$ amino acids) using only 20 metagenomic sequencing samples, and the strain dropout screen tested 10,712 interactions (104 × 103 strains) using only 104 sequencing samples. Candidate interactions were observed in the background of a community and are therefore more likely to be relevant under native conditions; many of them were validated in co-culture experiments (Figure 4E). Taken together, our system for community assembly and measurement establish a framework for rapid, robust experimentation with complex consortia.

In its current form, our approach has two important limitations. First, when propagated in vitro, our community exhibits a different architecture than is observed in vivo. The consortium was dominated by 374 Firmicutes that are typically found at lower relative abundances in the human gut, likely because the defined growth medium we used (SAAC) is rich in amino acids. Lowering the free amino acid content of the growth media and adding glycans, complex polypeptides, and host-derived factors such as bile acids could help steer the community toward a more typical in vivo architecture.

Second, the scale and complexity of our amino acid and strain dropout experiments precluded the possibility of carrying out multiple replicates. During the single passage over which communities were propagated, biomass increased by only 200 -fold. As a result, the interactions we identified likely include some false positives; replicate experiments and additional growth passages could yield a larger or higher confidence set of interactions. Nonetheless, the strain-amino acid and strain-strain interactions we 
383

384

385

386

387

388

389

390

391

392

393

394

395

396

397

398

399

400

401

402

403

404

405

406

407

408

409

410

411

412

413

414

416 C. sporogenes mutants are available on request. The strains used in this study are available from the 417 sources listed in the Key Resource Table.

validated show that the experiments were sufficiently sensitive and reproducible to uncover real interactions.

The properties of this community in the context of host colonization are described in an accompanying manuscript. Together, these efforts constitute a starting point for a defined, full-scale model system for the gut microbiome. Such a system would yield great dividends, as the research communities around yeast, worms, flies, and mice have shown over decades. It would be even more powerful in conjunction with new genetic tools to manipulate specific members of this community.

\section{STAR $\star M E T H O D S$}

Detailed Methods are provided in the online version of this paper and include the following:

- KEY RESOURCES TABLE

- RESOURCE AVAILABILITY

- Lead contact

- Materials availability

- Data and code availability

- EXPERIMENTAL MODEL AND SUBJECT DETAILS

- Bacterial strains and culture conditions

- METHOD DETAILS

- Metagenomic sequencing

- Metagenomic read mapping

- Amino-acid dropout experiments

- Creation of $C$. sporogenes mutants

- ATP assay

- Strain-dropout experiments

- Pairwise co-culture growth measurements

- QUANTIFICATION AND STATISTICAL ANALYSIS

\section{STAR $\star$ METHODS}

\section{Lead contact}

Further information and requests for resources and reagents should be directed to and will be fulfilled by the lead contact, Michael Fischbach (fischbach@fischbachgroup.org).

\section{Materials availability}




\section{Data and code availability}

420 Metagenomic and whole-genome sequencing datasets generated for this study will be available at the 421 Sequence Read Archive at the time of publication. Ninjamap is available at 422 https://github.com/FischbachLab/ninjamap and the associated docker containers are available at 423 https://hub.docker.com/orgs/fischbachlab/repositories.

\section{EXPERIMENTAL MODEL AND SUBJECT DETAILS}

\section{Bacterial strains and culture conditions}

Bacterial strains were selected based on metagenomic sequencing data from the NIH Human Microbiome Project (Kraal et al., 2014). The mean relative abundance and prevalence of each strain were quantified using the 81 samples from healthy human patients from North America. The $\sim 200$ strains that appeared in $\geq 37$ of the 81 samples were considered for inclusion in the community. We were able to obtain 104 of these strains from public repositories (Key Resources Table).

Strains were cultured in anaerobic conditions $\left(10 \% \mathrm{CO}_{2}, 5 \% \mathrm{H}_{2}, 85 \% \mathrm{~N}_{2}\right)$ in $2 \mathrm{~mL}$ 96-well plates for 24-48 $\mathrm{h}$ in their respective growth media (Table S1): Mega Medium (McNulty, et. al. 2015) supplemented with $400 \mu \mathrm{M}$ Vitamin K2, or Chopped Meat Medium supplemented with Mega Medium carbohydrate mix (McNulty, et. al. 2015) and $400 \mu \mathrm{M}$ Vitamin K2. For strain storage, $200 \mu \mathrm{L}$ of liquid culture was aliquoted $1: 1$ into sterile $50 \%$ glycerol in a $1 \mathrm{~mL} 96$-well plate. The plate was covered with an airtight silicone fitted plate mat, edges were sealed with $\mathrm{O}_{2}$-impervious yellow vinyl tape, and the plate was frozen at $-80^{\circ} \mathrm{C}$. To revive cultures, the storage plate was defrosted in the anaerobic chamber and $100 \mu \mathrm{L}$ from each well was used to inoculate $900 \mu \mathrm{L}$ of appropriate fresh medium. Twenty-four hours post-revival, each well was were used to monitor potential contamination.

\section{METHOD DETAILS}

\section{Constructing high quality genome assemblies}

We obtained the latest RefSeq (O'Leary et al., 2016) assembly for each strain in our community and assessed its quality based on contig statistics from Quast (Gurevich et al., 2013) v. 5.0.2 and SeqKit (Shen et al., 2016) v. 0.12.0, using GTDB-tk (Chaumeil et al., 2019) v. 1.2 .0 for taxonomic classification. A linear combination of the completeness and contamination scores (completeness $-5 \times$ contamination) derived from the CheckM (Parks et al., 2015) v. 1.1.2 lineage workflow was used along with the other metrics to include or exclude genomes in the GTDB (Parks et al., 2018, 2020) release 89 database (https://gtdb.ecogenomic.org/faq\#gtdb selection criteria). Genomes that contained any number of Ns, 
contained over 100 contigs, contained GTDB lineage warnings or multiple matches, or had CheckM completeness $<90$, contamination $>10$, and combination score $<90$ were resequenced and reassembled.

Our hybrid assembly pipeline contains a workflow for de novo and reference-guided genome assembly using both Illumina short reads and PacBio or Nanopore long reads. The workflow has three main steps: read pre-processing, hybrid assembly, and contig post-processing. Read pre-processing included 1) quality trimming/filtering (bbduk.sh adapterFile="adapters, phix" k=23, hdist=1, qtrim=rl, ktrim=r, entropy $=0.5$, entropywindow $=50$, entropyk=5, trimq $=25$, minlen=50), with adaptors and phix removed with kmer right trimming, kmer size of 23, Hamming distance 1 (allowing one mismatch), quality trimming of both sides of the read, filtering of reads with an average entropy $<0.5$ with entropy kmer length of 5 and a sliding window of 50, trimming to a Q25 quality score, and removal of reads with length $<50 \mathrm{bp} ; 2$ ) deduplication (bbdupe.sh); 3) coverage normalization (bbnorm.sh min=3) such that depth $<3 x$ was discarded; 4) error correction (tadpole.sh mode=correct); and 5) sampling (reformt.sh). All pre-processing was carried out using BBtools v. 38.37 for short reads. For long reads, we used filtlong v. 0.2 .0 (fitlong -min_length 1000 --keep_percent 90 --length_weight 10) to discard any read $<1 \mathrm{~kb}$ and the worst $10 \%$ of read bases, as well as to weigh read length as more important when choosing the best reads. Hybrid assembly was performed by Unicycler (Wick et al., 2017) v. 0.4 .8 with default parameters using preprocessed reads. After assembly, the contigs from the assembler were scaffolded by LRScaf (Qin et al., 2018) v. 1.1 .9 with default parameters. If the initial assembly did not produce the complete genome, gaps were filled by long reads TGS-GapCloser (Xu et al., 2019) v. 1.0.1 with default parameters. from the contaminated assembly using MetaBAT2 (Kang et al., 2019) v. 2.2.14 with default parameters.

Finally, the assembled genomes were evaluated using the same criteria as the RefSeq assemblies, and the assembly for each species with the best overall quality metrics was chosen as the reference assembly. This procedure resulted in the replacement of eight genomes: two from a PacBio/Illumina hybrid assembly, one from a Nanopore/Illumina hybrid assembly, one from a reference-guided Illumina assembly, and four from short-read assemblies of the respective isolate samples followed by binning (Table S5).

\section{Generating simulated sequencing reads}

In silico data were generated to evaluate the Ninjamap algorithm in the absence of genome assembly errors and sequencing quality issues. Grinder (Angly et al., 2012) v. 0.5.4 was applied to each genome to generate error-free reads with the following parameters: -read_distribution 140, -insert_size 487800 , -mate_orientation FR, -delete_chars '- *NX', -mutation_dist uniform 0, -random_seed 1712, - 
abundance_model uniform, -qual_levels 33 31, -fastq_output 1. The -coverage_fold parameter was adjusted based on the cases described below.

\section{Uniform abundance isolate dataset}

This dataset was created to test the sensitivity and specificity of the algorithm against our database of genomes. In silico data were generated for each genome with uniform coverage of 10X or 100X. We were able to consistently identify the correct genome regardless of coverage (Figure $2 \mathrm{C}$ ). Some cross mapping was observed at $\sim 0.01 \%$ relative abundance, likely because some genomes in our database shared more than $99 \%$ average nucleotide identity, making cross-mapping unavoidable. Thus, we generally treat $10^{-4}$ as a conservative lower bound for confident relative abundance estimation.

\section{Variable abundance community dataset}

In silico reads were generated for each genome at 10X, $0.1 \mathrm{X}$, and $0.001 \mathrm{X}$ uniform coverage. Three datasets of mixed community reads were generated including every genome at a coverage randomly selected from the three levels. The observed relative abundance of each genome in our database was calculated using the NinjaMap algorithm and compared to the expected relative abundance based on coverage level, which ranged from $\sim 3 \times 10^{-6}$ to 0.03 . We could estimate relative abundances accurately for genomes present at $>10^{-5}$ (Figure 2D). However, for lower relative abundances, we observed some discrepancies corresponding to the same genomes with mis-mapping against isolate datasets, indicating that high similarity between genomes begins to confound the algorithm at very low relative abundances.

\section{Metagenomic sequencing}

The same experimental pipeline was used for sequencing bacterial isolates and synthetic communities. Bacterial cells were pelleted by centrifugation under anaerobic conditions. Genomic DNA was extracted using the DNeasy PowerSoil HTP kit (Qiagen) and quantified in 384-well format using the Quant-iT PicoGreen dsDNA Assay Kit (Thermofisher). Sequencing libraries were generated in 384-well format using a custom low-volume protocol based on the Nextera XT process (Illumina). Briefly, the concentration of DNA from each sample was normalized to $0.18 \mathrm{ng} / \mu \mathrm{L}$ using a Mantis liquid handler (Formulatrix). If the concentration was $<0.18 \mathrm{ng} / \mu \mathrm{L}$, the sample was not diluted further. Tagmentation, neutralization, and PCR steps of the Nextera XT process were performed on a Mosquito HTS liquid handler (TTP Labtech), leading to a final volume of $4 \mu \mathrm{L}$ per library. During the PCR amplification step, custom 12bp dual unique indices were introduced to eliminate barcode switching, a phenomenon that occurs on Illumina sequencing platforms with patterned flow cells (Sinha et al., 2017). Libraries were pooled at the desired relative molar ratios and cleaned up using Ampure XP beads (Beckman) to achieve buffer removal and library size selection. The cleanup process was used to remove fragments $<300 \mathrm{bp}$ or $>1.5 \mathrm{kbp}$. Final 
523 library pools were quality-checked for size distribution and concentration using a Fragment Analyzer 524 (Agilent) and qPCR (BioRad). Sequencing reads were generated using a NovaSeq S4 flow cell or a 525 NextSeq High Output kit, in 2x150 bp configuration. 5-10 million paired-end reads were targeted for isolates 526 and 20-30 million paired-end reads for communities.

527

528

\section{Generating and normalizing the NinjaMap database}

529

The first step in the pipeline was to assess the uniqueness of each genome in the community. We 530 generated error-free in silico reads such that each genome was uniformly covered at 10x depth. Each such genome read set was aligned to all genomes in the community. The uniqueness of a genome was defined as the fraction of the genome that did not have reads cross-mapped from another strain; uniqueness values were between 0 and 1 , such that more unique genomes have a value closer to 1 . The uniqueness value of a strain was used to normalize its final relative abundance in any community sample. All genome sequences were combined into one fasta file and a Bowtie2 (Langmead and Salzberg, 2012) v. 2.3.5.1 index was computed for future alignments. The database and strain weights were recomputed each time the community or a genome was updated.

538

\section{Metagenomic read mapping}

Paired-end reads from each sample were aligned to the database using Bowtie2 with maximum insert length (-maxins) 3000, maximum alignments (-k) as 300, suppressed unpaired alignments (--nomixed), suppressed discordant alignments (--no-discordant), suppressed output for unaligned reads (--nounal), required global alignment (--end-to-end), and using the "--very-sensitive" alignment preset. The output was processed in Samtools (Li et al., 2009) to convert the alignment output from SAM output stream to BAM format. The BAM file was sorted and indexed by coordinates.

\section{NinjaMap alignment scoring}

A primary goal of the NinjaMap algorithm is to analyze and tabulate every input read. A successful match was defined as a read aligned to a genome at $100 \%$ identity across $100 \%$ of the read length. If a read was uniquely matched to a single strain, its mate pair was also recruited as long as it had at least one match to the same strain. If exactly 1 strain was a perfect match for both reads, the pair was considered a "primary pair" and a score of 1 was given for each read. If $>1$ or 0 strains were a match for both reads, both reads were placed in escrow and analyzed separately as described below.

By prioritizing paired-read scoring, noise was significantly reduced while ensuring that as many reads as possible were considered for abundance estimates. Once preliminary strain abundances were calculated based on primary pairs, reads in escrow were then assigned fractionally to the strains to which 
of each strain, normalized by the size of the unique region of each genome within the database, such that the total contribution for a read was 1. In some cases, an individual escrowed read matched to a strain without any matches to primary pairs; such reads were discarded and not used in the final estimates.

Finally, the total score for each strain in the database was normalized by the number of reads that aligned to the database, so that the relative abundances of all strains summed to 1 .

\section{Amino acid dropout experiments}

Strains were passaged by diluting 1:10 into fresh growth medium every $24 \mathrm{~h}$ for 2-3 days. The day before amino acid dropout experiments, cultures were diluted 1:10 into $1 \mathrm{~mL}$ of fresh medium and grown for $24 \mathrm{~h}$ as inoculation working stocks. To measure $\mathrm{OD}_{600}$, strains were diluted 1:10 into $150 \mu \mathrm{L}$ of the appropriate culture medium and a plate reader was used to measure absorbance at $600 \mathrm{~nm}$. Stocks were diluted to a final $O D_{600}$ of 0.1 using fresh growth medium. If a culture did not reach $O D_{600}$ of 0.1 , the entire culture was used as the working stock for community assembly. Equal volumes of each stock were pooled to create a 104-member synthetic community. The community was centrifuged at $5000 \times \mathrm{g}$ for $5 \mathrm{~min}$, washed, and resuspended in an equivalent volume of PBS to generate the pooled community working stock. SAAC medium (Dodd et al., 2017) was made containing all amino acids at $1 \mathrm{mM}$ concentration except for cysteine, which was added at $4.126 \mathrm{mM}$ (Table S6). Twenty similar media were made in which one amino acid at a time was removed. $1.6 \mathrm{~mL}$ of each medium was aliquoted in triplicate and inoculated with the pooled community at 1:10 or 1:100 dilution. Four $100 \mu \mathrm{L}$ aliquots of each culture were collected at $48 \mathrm{~h}$ and processed for metagenomic sequencing.

\section{Constructing $C$. sporogenes mutants}

C. sporogenes deletion mutants were constructed using a previously reported protocol (Guo et al., 2019); the strains and primers used for each mutant are listed in Table S7. In brief, from plasmids CS_OTC and CS_ADI—which harbor the targeting and repair templates-we amplified DNA sequences encoding the gRNA locus (the gRNA plus adjacent elements and the repair template) and ligated the amplicon into the pMTL82254 backbone. These repair templates consist of 700- to 1200-bp sequences flanking the 40to 100-bp sequence targeted for excision.

To construct the $\triangle$ adi strain, a gRNA fragment was purchased from Quintara and amplified with primers fwd_pMTL82254_Notl, rev_gRNA_flank1. The two flanking regions were amplified from C. sporogenes genomic DNA using the primers 5rev_flank1 and 5fwd_flank1_flank2 for flank 1 and 5rev_flank1_flank2 and 5fwd_flank1_flank2 for flank 2 . Next, the flanking regions were joined by amplifying with primers fwd_gRNA_flank1 and rev_flank2. The amplified gRNA fragment was attached to the joined flank construct by amplifying with primers fwd_pMTL82254_Notl and rev_pMTL82254_Ascl. Finally, the 
592 pMTL82254 plasmid and the construct containing the gRNA, flank1, and flank2 regions were digested with 593 Notl and Ascl and ligated with T4 ligase (NEB). The final construct was named CS_ADI.

$594 \quad$ To make the $\Delta$ otc strain, the gRNA fragment was purchased from Quintara and amplified with 595 fwd_pMTL82254_Notl and rev_OTC_gRNA_flank1. The two flanking regions were amplified from C. 596 sporogenes genomic DNA using the primers fwd_OTC_gRNA_flank1 and rev_OTC_flank1_flank2 for flank 5971 and fwd_OTC_flank1_flank2 and rev_OTC_flank2 for flank 2. Next, the flanking regions were joined by 598 amplifying with the primers fwd_OTC_gRNA_flank1 and rev_OTC_flank2. The amplified gRNA fragment 599 was attached to the joined flank construct by amplifying with fwd_pMTL82254_Notl and 600 rev_pMTL82254_Ascl. Finally, the pMTL82254 plasmid and the construct containing the gRNA, flank1, 601 and flank2 regions were digested with Notl and Ascl and ligated with T4 ligase (NEB). The final construct 602 was named CS_OTC.

603 CS_OTC or CS_ADI was electroporated into Escherichia coli S17 cells and conjugated into C. 604 sporogenes strain ATCC 15579 using a previously described method (Guo et al., 2019). In brief, a single 605 colony of wild-type C. sporogenes was used to inoculate $2 \mathrm{~mL}$ of TYG broth (3\% (w/v) tryptone, 2\% (w/v) 606 yeast extract, $0.1 \%(\mathrm{w} / \mathrm{v})$ sodium thioglycolate) and incubated anaerobically in an atmosphere consisting 607 of $10 \% \mathrm{CO}_{2}, 5 \% \mathrm{H}_{2}$, and $85 \% \mathrm{~N}_{2}$. E. coli S17 cells with CS_OTC or CS_ADI were grown in LB broth 608 supplemented with $250 \mu \mathrm{g} / \mathrm{mL}$ erythromycin at $30^{\circ} \mathrm{C}$ with shaking at $225 \mathrm{rpm}$. After $17-24 \mathrm{~h}, 1 \mathrm{~mL}$ of this 609 culture was centrifuged at $1000 \times g$ for $1 \mathrm{~min}$ and washed twice with $500 \mu \mathrm{L}$ of PBS (40 mM potassium 610 phosphate, $10 \mathrm{mM}$ magnesium sulfate, $\mathrm{pH}$ 7.2). The pellet was transferred into the anaerobic chamber 611 and $250 \mu \mathrm{L}$ of $C$. sporogenes overnight culture was added and mixed with the cell pellet. $30 \mu \mathrm{L}$ aliquots of 612 the mixture were plated on a pre-reduced TYG agar plate in eight spots. The plate was tilted to coalesce 613 the spots and incubated for $24 \mathrm{~h}$. Biomass from the plate was scraped using a sterile inoculation loop and 614 suspended in $250 \mu \mathrm{L}$ of pre-reduced PBS. $100 \mu \mathrm{L}$ of the cell suspension was plated on TYG agar 615 containing $10 \mu \mathrm{g} / \mathrm{mL}$ erythromycin and $250 \mu \mathrm{g} / \mathrm{mL}$ D-cycloserine to isolate single colonies. One colony was 616 picked, sequence verified, and used as the starting point for the next conjugation.

In the second conjugation, E. coli S17 cells containing pMTL83153_fdx_Cas9 were grown in LB 618 619 620 621 622 623 broth supplemented with $25 \mu \mathrm{g} / \mathrm{mL}$ chloramphenicol at $30{ }^{\circ} \mathrm{C}$ with shaking at $225 \mathrm{rpm}$. After washing, the pellet was moved into the anaerobic chamber and $250 \mu \mathrm{L}$ of an overnight culture of $C$. sporogenes harboring the CS_OTC vector was thoroughly mixed with the E. coli cell pellet. $30 \mu \mathrm{L}$ aliquots of the mixture was plated on a pre-reduced TYG agar plate in eight spots. The plate was tilted to coalesce the spots and incubated for $72 \mathrm{~h}$. Biomass from the plate was scraped using a sterile inoculation loop and resuspended in $250 \mu \mathrm{L}$ of pre-reduced PBS. $100 \mu \mathrm{L}$ of the cell suspension was plated on each of two pre-reduced TYG agar plates containing $10 \mu \mathrm{g} / \mathrm{mL}$ erythromycin, $15 \mu \mathrm{g} / \mathrm{mL}$ thiamphenicol, and $250 \mu \mathrm{g} / \mathrm{mL}$ D-cycloserine. $C$. sporogenes colonies typically appeared after 36-48 h, and 8-10 colonies were re-streaked on pre-reduced TYG agar plates containing $10 \mu \mathrm{g} / \mathrm{mL}$ erythromycin, $15 \mu \mathrm{g} / \mathrm{mL}$ thiamphenicol, and $250 \mu \mathrm{g} / \mathrm{mL}$ D-cycloserine 
627 to isolate single colonies. The isolated colonies were used to inoculate pre-reduced TYG broth 628 supplemented with $10 \mu \mathrm{g} / \mathrm{mL}$ erythromycin and $15 \mu \mathrm{g} / \mathrm{mL}$ thiamphenicol, and genomic DNA was isolated 629 using a Quick DNA fungal/bacterial kit (Zymo Research). Primers ADI_532_fwd and ADI_22_rev or 630 OTC_5_up_fwd and OTC_930_down_rev (Table S2) were used to verify deletions.

\section{ATP assay}

An aliquot from a frozen stock of $C$. sporogenes was used to inoculate $5 \mathrm{~mL}$ of TYG broth and grown to stationary phase ( $24 \mathrm{~h})$. Cells were diluted 1:1000 into $20 \mathrm{~mL}$ of TYG broth and grown to latelog phase $(\sim 16 \mathrm{~h})$. Cells were harvested by centrifugation $\left(5,000 \times \mathrm{g}\right.$ for $10 \mathrm{~min}$ at $\left.4{ }^{\circ} \mathrm{C}\right)$ and washed twice with $20 \mathrm{~mL}$ of pre-reduced PBS. $100 \mu \mathrm{L}$ of cells was seeded into rows of a 96-well microtiter plate (12 wells per condition). $200 \mu \mathrm{L}$ of pre-reduced $2 \mathrm{mM}$ substrate (arginine) in phosphate washing buffer, or $200 \mu \mathrm{L}$ of buffer alone, were dispensed into rows of a separate 96-well microplate. At $t=0,100 \mu \mathrm{L}$ of substrate or buffer were added to the cells and mixed gently by pipetting. At $t=-5 \mathrm{~min},-1 \mathrm{~min}, 30 \mathrm{~s}, 1 \mathrm{~min}, 2 \mathrm{~min}, 5 \mathrm{~min}$, $10 \mathrm{~min}, 20 \mathrm{~min}, 30 \mathrm{~min}, 45 \mathrm{~min}, 60 \mathrm{~min}$, and $90 \mathrm{~min}, 10 \mu \mathrm{L}$ of cells were extracted and mixed with $90 \mu \mathrm{L}$ of DMSO to quench the reaction and liberate cellular ATP. For the time points $t=-5$ min and -1 min-prior to the addition of buffer or substrate $-5 \mu \mathrm{L}$ of cell suspension was harvested and $5 \mu \mathrm{L}$ of either buffer or substrate were added to the cell-DMSO mixture to bring the total volume to $100 \mu \mathrm{L}$. The ATP content from $10 \mu \mathrm{L}$ aliquots of lysed cells was measured using a luminescence-based ATP determination kit (Invitrogen, Cat. \#A22066). Absolute ATP levels were calculated using a calibration curve with known concentrations of ATP.

647

\section{Strain dropout experiments}

649

Strains were passaged by diluting 1:10 into fresh growth medium every $24 \mathrm{~h}$ for 2-3 days. The day 650 before strain dropout experiments, cultures were diluted 1:10 into $1 \mathrm{~mL}$ of medium and grown overnight as inoculation working stocks. To measure $\mathrm{OD}_{600}$, strains were diluted 1:10 into $150 \mu \mathrm{L}$ of the appropriate culture medium and a plate reader was used to measure absorbance at $600 \mathrm{~nm}$. Stocks were normalized using fresh growth medium to a final $O D_{600}$ of 0.1 . If an overnight culture did not reach $\mathrm{OD}_{600}$ of 0.1 , the entire culture was used as the working stock for community assembly. For community assembly, $10 \mathrm{~mL}$ of each stock were pipetted into a 12-well reservoir plate.

Strain dropouts were performed on a row-by-row basis in a 96-well deep-well plate. For example, to individually dropout each of the 12 strains in row $A$, equal volumes of all community members not in row A were pooled and aliquoted into 12 sterile $1.5 \mathrm{~mL}$ Eppendorf tubes. This process resulted in $120 \mu \mathrm{L}$ of each of 104-12=92 strains for each of 12 communities being combined into a $11.04 \mathrm{~mL}$ pool, which was divided into 12 aliquots of $920 \mu \mathrm{L}$ in sterile Eppendorf tubes. To add the strains in row A, 12 sub- 
$662 \mu \mathrm{L}$ of PBS were added, for a total volume of $120 \mu \mathrm{L}$, which was added to the $920 \mu \mathrm{L}$ pooled community 663 lacking row $A$, resulting in the creation of 12 communities with volume $1040 \mu \mathrm{L}$, each lacking one of the 664104 strains. This process was repeated for all rows to cover the 104 strains over 2 days; 24 and 80 dropouts 665 were constructed on day 1 and 2, respectively. Each dropout community was washed once via 666 centrifugation at $5000 \times g$ for $5 \mathrm{~min}$ and resuspended in an equal volume of sterile PBS. Sixteen microliters of each dropout community were used to inoculate $1.6 \mathrm{~mL}$ of SAAC medium. Four hundred microliter 668 aliquots were collected at $12 \mathrm{~h}, 24 \mathrm{~h}$, and $48 \mathrm{~h}$ post-inoculation. The initial dropout community stocks and all aliquots were processed for metagenomic sequencing using a Power Soil DNA extraction kit (Qiagen).

\section{Pairwise co-culture growth measurements}

Each strain was inoculated from a frozen stock into its optimal growth medium (Mega Medium or Chopped Meat Medium) and the culture was incubated for $24 \mathrm{~h}$ at $37^{\circ} \mathrm{C}$. $\mathrm{OD}_{600}$ was measured, and $1 \mathrm{~mL}$ of each strain was washed with sterile 1x PBS. $0.75 \mu \mathrm{L}$ of each strain (for co-cultures) or PBS (for monocultures) was added to $148.5 \mu \mathrm{L}$ SAAC medium for a total volume of $150 \mu \mathrm{L}$. The baseline $\mathrm{OD}_{600}$ and growth curves were measured using an Epoch plate reader (Biotek). For C. sporogenes and L. lactis, cultures were normalized to $\mathrm{OD}_{600}=1.0$ before mixing. All cultures were performed in technical triplicate with 2-6 biological replicates. For each growth curve, the interaction score $\alpha$ was computed based on an additive null model (Aranda-Díaz et al., 2020):

$$
\alpha=\frac{\mathrm{OD}_{\mathrm{co}}-\left(\mathrm{OD}_{\mathrm{s} 1}+\mathrm{OD}_{\mathrm{s} 2}\right)}{\sqrt{\mathrm{OD}_{\mathrm{s} 1} \mathrm{OD}_{\mathrm{s} 2}}}
$$

where $O D_{c 0}, O D_{s 1}$, and $O D_{s 2}$ are the maximum $O D$ values of the co-culture and the individual strains $s 1$ and $s 2$, respectively. The time to reach half maximum absorbance $\left(t_{1 / 2}\right)$ was measured for individual cultures and co-cultures and labeled as significant if average co-culture $t_{1 / 2}$ was greater than either individual average $t_{1 / 2}$. Student's t-test was utilized to determine significant changes with $p<0.05$.

\section{Mis-mapping estimation using monoculture sequencing}

Read fractions were analyzed using custom Matlab (Mathworks, R2018a) code. Read fractions were rescaled to sum to 1 , thereby reflecting the relative abundances of reads mapped to one of the 104

$$
E_{i, j}=\sum_{i \neq k} R_{k, j} \times r_{i, k}
$$


693 where $R_{k, j}$ is the relative abundance of strain $k$ in sample $j$ and $r_{i, k}$ is the relative abundance of strain $i$ in 694 sequencing data of strain $k$ as an isolate. Strains whose relative abundance was comparable to the 695 expected mis-mapped relative abundance $\left(E_{i, j}<10 r_{i, j}\right)$ were removed from further analysis.

\section{Amino acid dropout data analysis}

Read fractions were rescaled to sum to 1 , thereby reflecting the relative abundances of reads mapped to one of the 104 genomes in our database. The effect of removal of an amino acid on a strain

was estimated by calculating the $z$ score $z_{k, j}=\frac{R_{k, j}-\mu_{k}}{\sigma_{k}}$, where $R_{k, j}$ is the $\log _{10}$ (relative abundance) of strain 701 $k$ in sample $j$ and $\mu_{k}$ and $\sigma_{k}$ are the mean and standard deviation, respectively, of $\log _{10}$ (relative abundance) 702 for strain $k$ across all samples except the cysteine dropout. The cysteine dropout sample was excluded 703 from the calculation of $\mu_{k}$ and $s_{k}$ because this sample was an obvious outlier. Data points that could be 704 explained by mismapping were removed. Putative interactions were identified based on $\left|z_{j, k}\right|>2$, i.e. amino 705 acid dropouts that changed the $\log _{10}$ (relative abundance) of strain $k$ by $\geq 2$ standard deviations relative to 706 its mean. Some strains varied in relative abundance by several orders of magnitude; as a result, $\sigma_{k}$ was 707 large, so putative interactions would be missed using z-scores.

708

To identify clusters of strains that responded similarly or amino acids that elicited a similar 709 response, we normalized $R_{k, j}$ for each strain across samples by subtracting $\mu_{k}$ and performed hierarchical 710 clustering of both strains and amino acid dropouts on a dataset including strains that were detected in all 21 amino acid dropout samples.

712

713

\section{Strain interactions in strain-dropout data}

714

Read fractions were rescaled to sum to 1 , reflecting the relative abundances of reads mapped to 715 one of the 104 genomes in our database. Samples with $<10^{6}$ reads, as well as the Eubacterium siraeum DSM 15702 dropout (which appeared to be missing an additional strain, $A$. caccae), were discarded from further analysis.

To identify batch effects that caused unintentional groupings within the dataset, we subtracted the mean $\log _{10}$ (relative abundance) of each strain in $t=48 \mathrm{~h}$ samples from the $\log _{10}$ (relative abundance) in each sample. These normalized relative abundances were used to calculate the Pearson correlation coefficient of each pair of samples. Based on this correlation matrix, samples were split into 4 experimental groups that corresponded to natural groupings arising from the experimental setup (Figure S6), with group sizes $n=21,57,12$ and 8. For the two largest groups, z-scores were calculated for each strain based on the mean and standard deviation of $\log _{10}$ (relative abundances) within the corresponding experimental group. To account for noise in low-abundance strains, z-scores were only calculated for strains that were undetected in $<5$ samples in the experimental group. Data points that could be explained by mismapping 
728

729

730

731

732

733

734

735

736

737

738

739

740

741

742

743

744

745

746

747

748

749

750

751

752

753

754

755

756

757

758

759

were only set to an arbitrary small number for graphical representation. Putative interactions were defined based on $\left|z_{j, k}\right|>2$.

\section{Statistical analysis}

The statistical details of experiments can be found in the figure legends. Reported $n$ values are the total samples (cultures) per group. Unless otherwise stated, $p$-values were not corrected for multiple hypothesis testing. Benjamini-Hochberg corrections, hypergeometric tests, Student's t-tests (unpaired or two-tailed), and Kruskal-Wallis tests were performed in MATLAB.

\section{ACKNOWLEDGMENTS}

We are deeply indebted to members of the Fischbach and Huang labs for helpful discussions, and to Rod Mackie (UIUC) for bacterial strains used in this study. A.A.-D. is a Howard Hughes Medical Institute International Student Research fellow, a Stanford Bio-X Bowes fellow, and a Siebel Scholar. This work was supported by the Stanford Microbiome Therapies Initiative (M.A.F., K.C.H.), NIH grants RM1 GM135102 (K.C.H.), DP1 DK113598 (M.A.F.), P01 HL147823 (M.A.F.), and R01 DK101674 (M.A.F.), the Bill and Melinda Gates Foundation (M.A.F.), an HHMI-Simons Faculty Scholars Award (M.A.F.), the Leducq Foundation (M.A.F.), the Stanford-Coulter Translational Research Grants Program (M.A.F.), the Chan Zuckerberg Biohub (K.C.H., M.A.F.), and the Allen Discovery Center at Stanford on Systems Modeling of Infection (K.C.H.).

\section{AUTHOR CONTRIBUTIONS}

Conceptualization: A.C., M.A.F. Methodology and investigation: A.C., A.A.-D., S.J., F.Y., M.I., X.M., A.P., A.W., A.L.S., A.D., K.C.H., M.A.F. Formal analysis: A.C., A.A.-D., S.J., F.Y., M.I., X.M., K.C.H., M.A.F. Visualization: A.C., A.A.-D., S.J., K.C.H., M.A.F. Supervision: K.C.H., M.A.F. Writing: A.C., A.A.-D., S.J., F.Y., K.C.H., M.A.F. All authors reviewed the manuscript before submission.

\section{DECLARATION OF INTERESTS}

Stanford University and the Chan Zuckerberg Biohub have patents pending for microbiome technologies on which the authors are co-inventors. M.A.F. is a co-founder and director of Federation Bio and Viralogic, a co-founder of Revolution Medicines, and a member of the scientific advisory boards of NGM Bio and Zymergen. A.G.C. has been a paid consultant to Federation Bio. All of the other authors have no competing interests. 
bioRxiv preprint doi: https://doi.org/10.1101/2021.06.15.448618; this version posted June 16, 2021. The copyright holder for this preprint (which was not certified by peer review) is the author/funder. All rights reserved. No reuse allowed without permission.

Prevalence

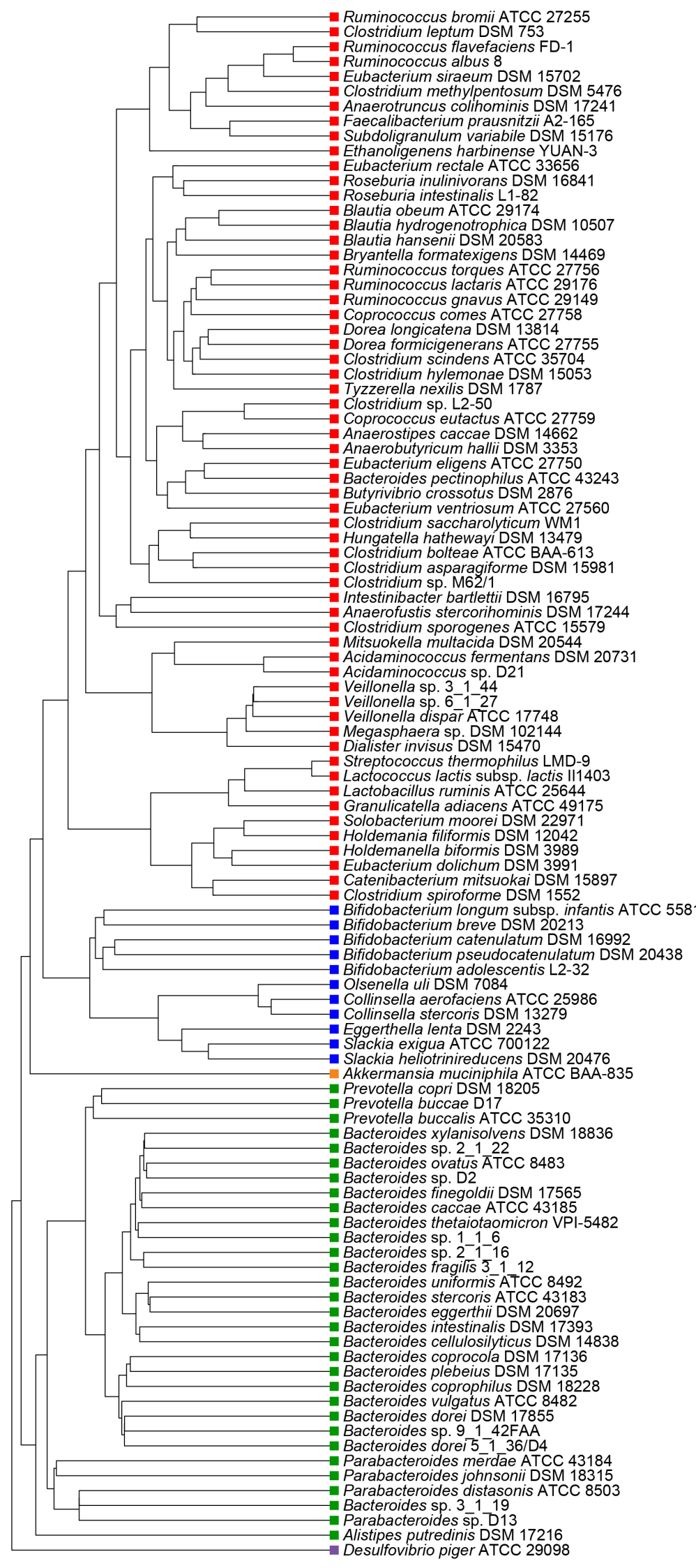

$\log _{10}$ (rel. abundance) mean \pm s.d.

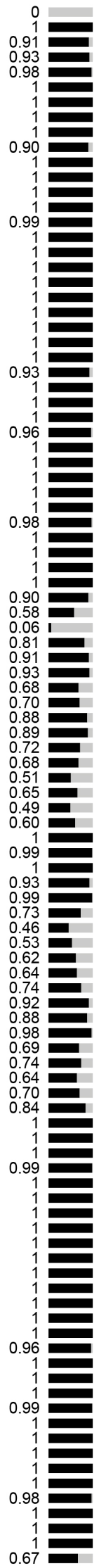

$-1.44 \pm 0.97$

$-2.94 \pm 0.87$

$-1.61 \pm 1.53$

$-1.42 \pm 0.66$

$\begin{aligned}-1.23 & \pm 0.78 \\ -3.29 & \pm 0.64\end{aligned}$

$-0.52 \pm 0.65$

$-1.05 \pm 0.76$

$-2.56 \pm 0.76$

$-2.60 \pm 0.71$

$-1.02 \pm 1.11$

$-1.36 \pm 0.74$

$-0.90 \pm 0.83$

$-1.08 \pm 0.82$

$-2.32 \pm 0.67$

$-3.40 \pm 0.61$

$-1.57 \pm 0.89$

$-3.17 \pm 0.61$

$-1.52 \pm 0.83$

$-0.62 \pm 0.91$

$-1.71 \pm 0.63$

$-1.05 \pm 0.80$

$-2.35 \pm 0.76$

$-2.36 \pm 0.75$

$-1.45 \pm 0.66$
$-2.89 \pm 0.87$

$-3.74 \pm 0.60$

$-4.04 \pm 0.57$
$-3.63 \pm 0.57$

$-3.50 \pm 0.56$

$-2.96 \pm 1.27$

$-3.50 \pm 0.99$

$-3.12 \pm 1.03$

$-3.50 \pm 0.59$
$-0.97 \pm 1.78$

$-3.06 \pm 0.95$

$-3.66 \pm 0.89$

$-3.50 \pm 0.71$
$-4.11 \pm 0.56$

$-3.69 \pm 0.77$

$-1.91 \pm 0.77$
$-2.53 \pm 0.84$

$-2.53 \pm 0.84$
$-2.34 \pm 0.68$

$-2.11 \pm 1.10$

$-3.58 \pm 0.84$

$-3.01 \pm 1.00$

$-2.80 \pm 1.03$

$-2.18 \pm 1.28$

$-1.53 \pm 1.28$
$-3.38 \pm 0.89$

$-2.93 \pm 0.83$

$-3.85 \pm 0.49$
$-3.79 \pm 0.47$

$-2.06 \pm 1.50$
$-3.06 \pm 1.92$

$-3.10 \pm 0.68$

$-2.29 \pm 0.50$

$-0.63 \pm 0.70$

$-0.59 \pm 0.70$

$-0.06 \pm 0.58$

$-0.66 \pm 0.76$

$-0.44 \pm 1.28$

$-0.23 \pm 0.70$

$-1.13 \pm 0.81$

$-1.43 \pm 09$
0.09
-0.82

$-0.16 \pm 1.13$

$-1.08 \pm 0.87$

$-1.05 \pm 0.71$

$-0.94 \pm 0.62$

$-0.99 \pm 0.59$

$-1.14 \pm 0.44 \pm 0.64$
0.44

$-0.29 \pm 0.62$

$-0.14 \pm 0.61$

$-0.20 \pm 1.23$

$-1.42 \pm 0.82$

$-0.86 \pm 1.02$

$-0.68 \pm 0.75$

$0.41 \pm 0.95$
$-3.32 \pm 1.03$

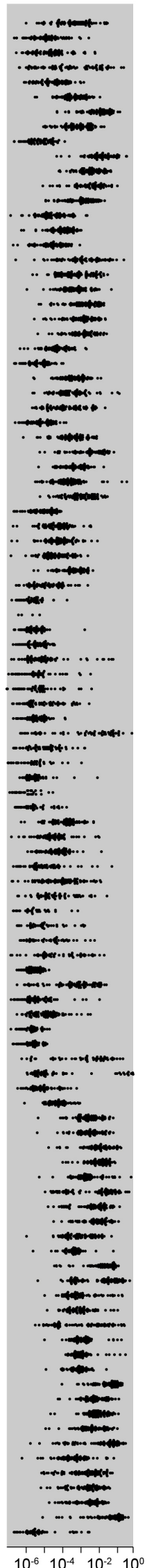

$-0.12 \pm 0.73$

$-3.29 \pm 0.64$
$0.08 \pm 0.69$
Genome size

(Mb)

2.15

4.5

(6.66

3.48

3.25

3.01
3.45
4.45

.05

4.41
3.63
3.63

3.63
3.06
4.55

2.74

2.74
2.73
3.50

3.24

3.24
3.19

3.19

3.89

2.95

2.95
3.10

3.61
3.29

2.83

3.03
2.50
2.87

4.66
7.16

7.16
6.56

6.42
3.84

2.97
2.28

4.10
2.58

2.33

2.24
2.16

2.17
2.12

2.50
1.90

1.86
2.37

2.11

1.95
2.34

3.93
2.52 
760 Figure 1: A complex gut bacterial community. A phylogenetic tree of the 104 strains in the community 761 based on a multiple sequence alignment of conserved single-copy genes. The community was designed 762 by identifying the most prevalent strains in sequencing data from the NIH Human Microbiome Project 763 (HMP). Colors indicate the phylum of each strain: Firmicutes = red, Actinobacteria = blue, Verrucomicrobia $764=$ orange, Bacteroidetes = green, and Proteobacteria = purple. Also shown are the prevalence and relative 765 abundance of each strain in the data set from the NIH HMP ( $n=81$ subjects), and the size of each strain's 766 genome. 
A

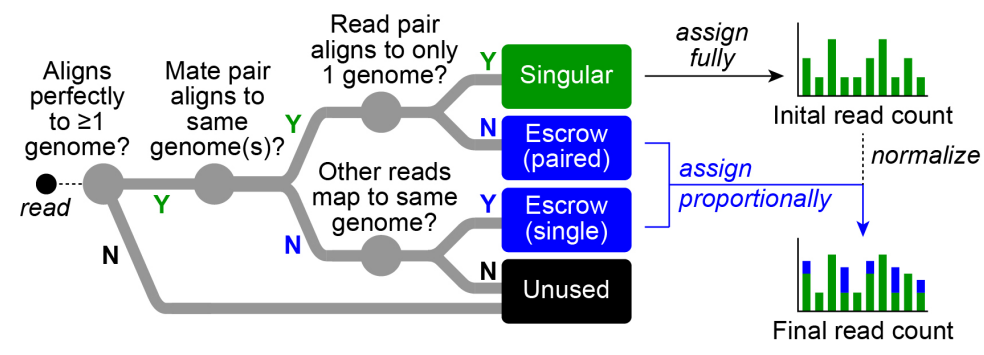

B

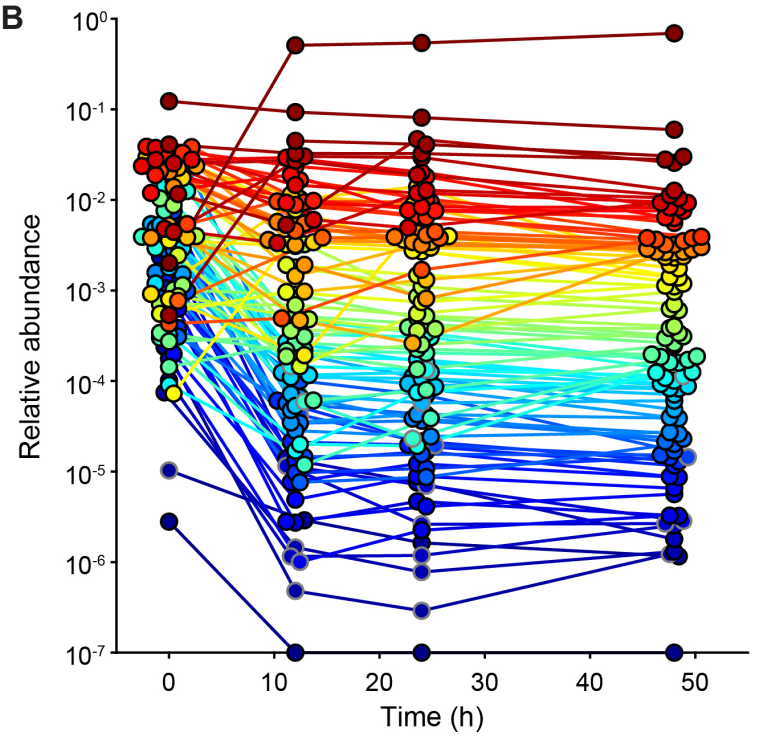

C

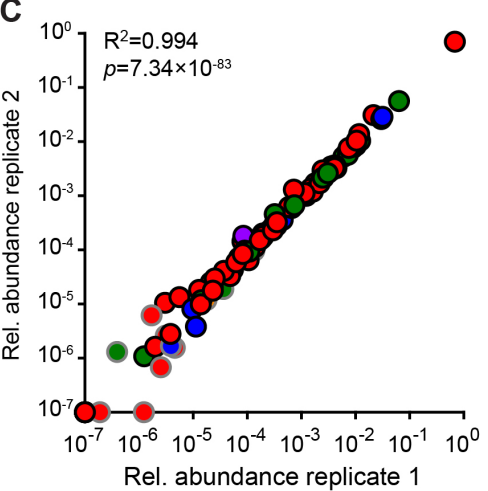

NinjaMap read fate
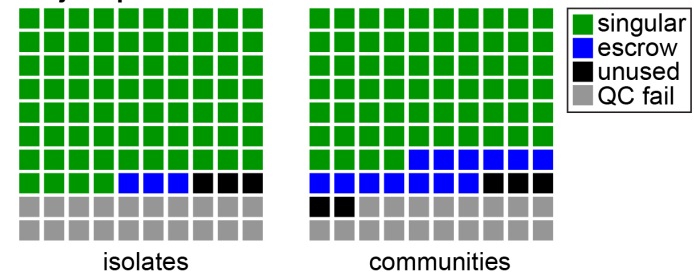

767

768

769

770

771

772

773

774

775

776

777

778

779

780

Figure 2: A sensitive and specific read mapping pipeline. (A) A schematic of NinjaMap, a new algorithm that quantifies strain abundances in defined communities with high accuracy. Reads that match a single genome unambiguously are assigned to that genome; reads that match multiple genomes are placed in escrow. An initial estimate of the relative abundance of each strain is computed from the unambiguous alignments and used to assign escrow reads proportionally. The final read counts are then normalized to obtain relative abundances. (B) The community reaches a stable configuration quickly. Each dot is an individual strain; the collection of dots in a column represents the community at a single timepoint. Strains are colored according to their rank-order abundance in the community at $48 \mathrm{~h}$. By $12 \mathrm{~h}$, The relative abundances of strains in the community span six orders of magnitude and remain largely stable through $48 \mathrm{~h}$. (C) Communities generated from the same inoculum (i.e., technical replicates) have a nearly identical composition at 48 h. (D) Communities generated from two inocula prepared on different days (i.e., biological replicates) have a similar architecture at $48 \mathrm{~h}$. In (C) and (D), the color of each circle represents the phylum of the corresponding species, and circles with gray outlines represent strains whose presence could be explained by read mis-mapping. 
A

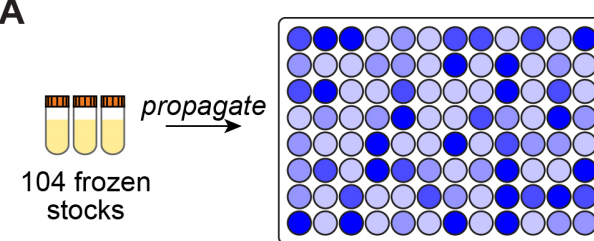

unnormalized cultures

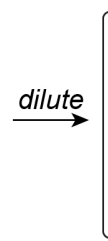

8

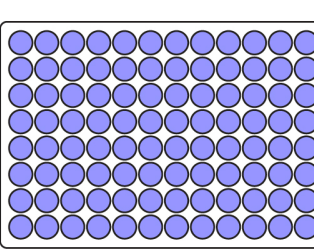

normalized cultures

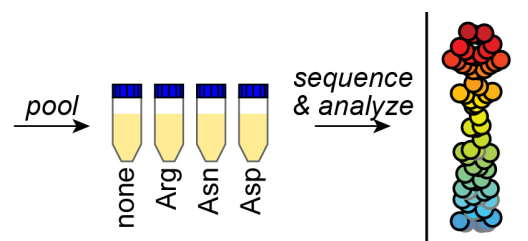

relative abundances

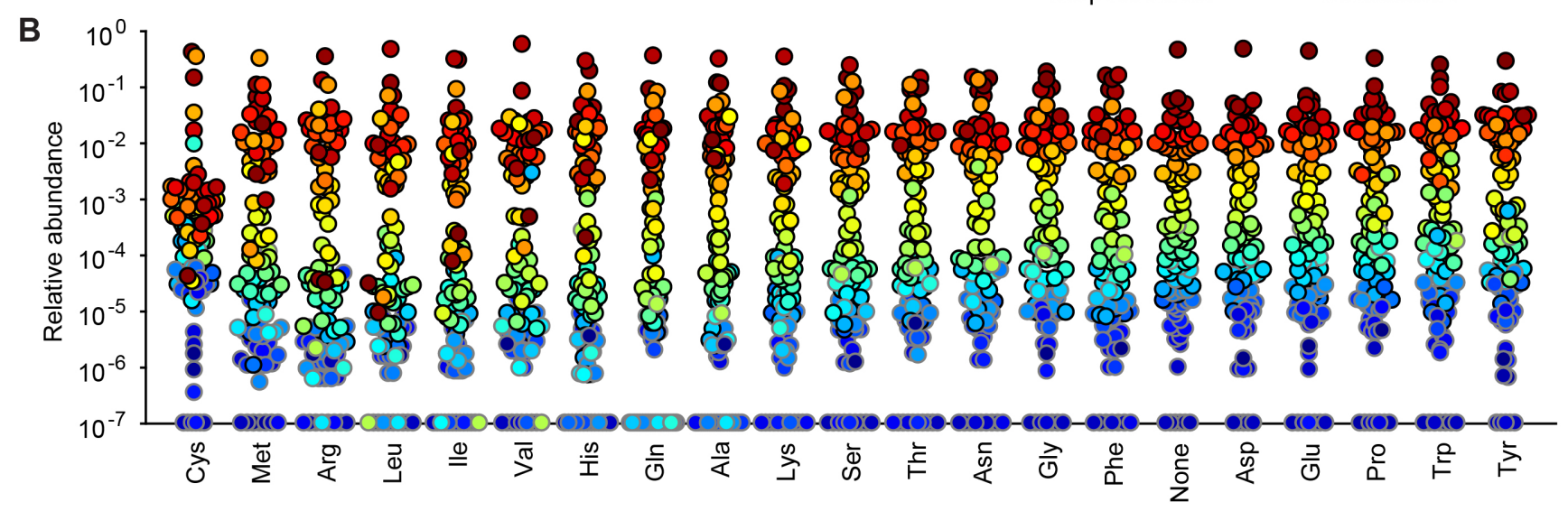

C

C. sporogenes ATCC

$L$. ruminis ATCC 25644

$P$. distasonis ATCC 8503

B. finegoldii DSM 17565

B. thetaiotaomicron VPI-5482

B. stercoris ATCC 43183

C. hylemonae DSM 15053

A. caccae DSM 14662

A. fermentans DSM 20731

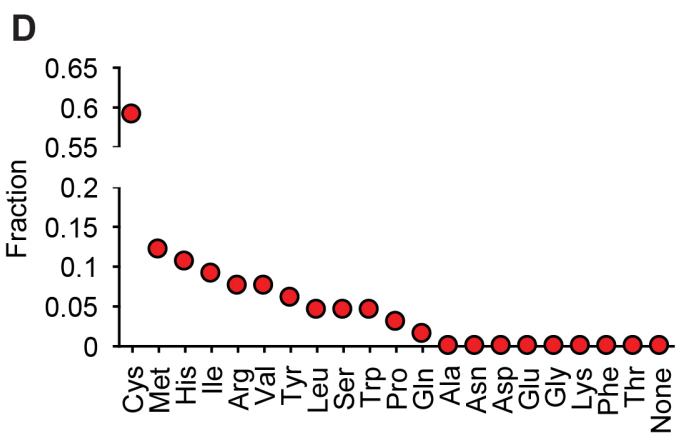

E

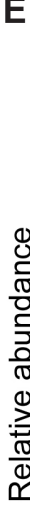

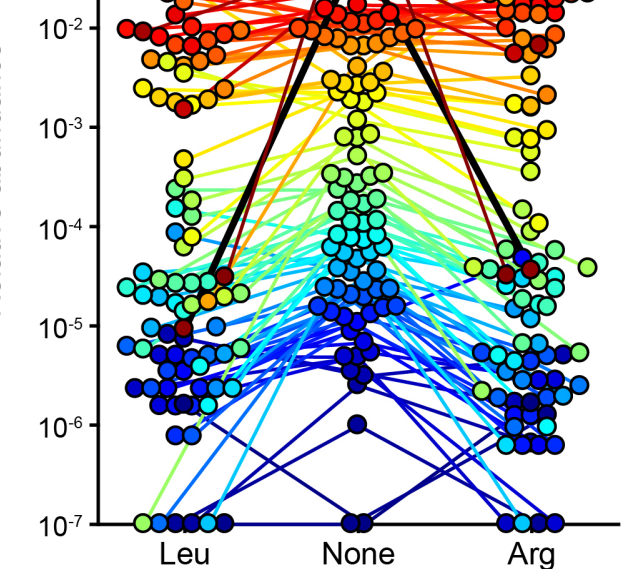

$\mathbf{F}$

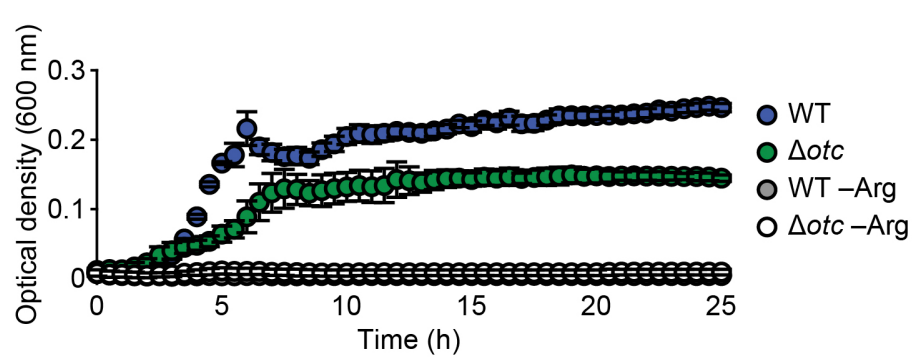

G

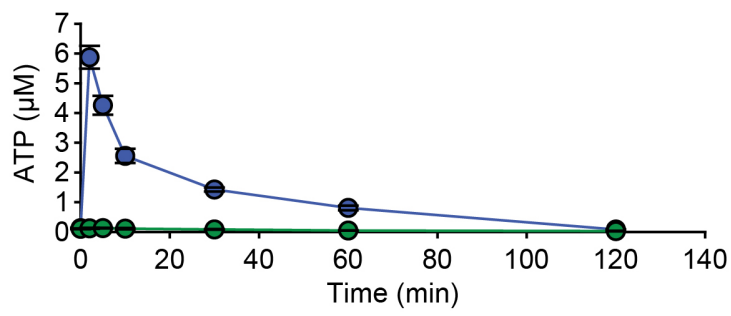

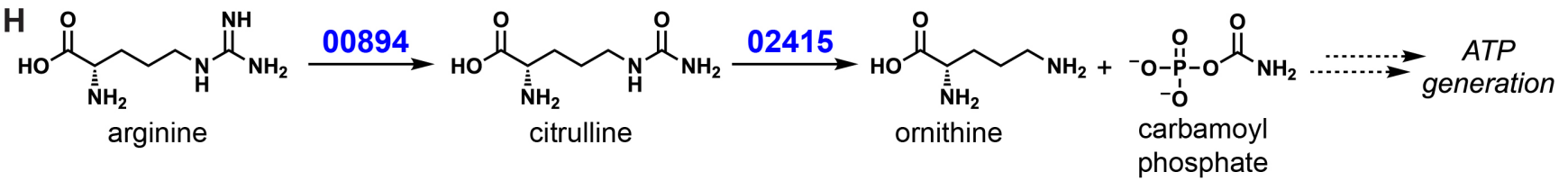


781 Figure 3: Systematic analysis of strain-amino acid interactions. (A) Schematic of the amino acid 782 dropout experiment. Frozen stocks of the 104 strains were used to inoculate cultures that were grown for $78324 \mathrm{~h}$, diluted to similar optical densities (to the extent possible), and pooled. The mixed culture was used 784 to inoculate one of twenty defined media lacking one amino acid at a time. After $48 \mathrm{~h}$, communities were 785 sequenced and analyzed by NinjaMap to determine changes relative to growth in the complete defined 786 medium. (B) Community composition is impacted by amino acid dropout. Each dot is an individual strain; 787 the collection of dots in a column represents the community at a single timepoint. Strains are colored 788 according to their rank-order abundance in the community grown in complete defined medium. Strains 789 whose relative abundance could be explained by read mis-mapping from a more abundant strain in the 790 same sample are plotted with a gray outline. Undetected strains were set to $10^{-7}$ for visualization. (C) A 791 heat map showing the $\log _{10}$ (relative abundance) normalized to strain mean for a subset of strains (full set 792 is shown Figure S3A). The Firmicutes L. lactis, C. sporogenes, and L. ruminis grow less robustly in the 793 absence of Leu and Ile. (D) The effect of amino acid removal varies widely across amino acids. A z-score 794 was calculated based on the standard deviation of strain abundance across all samples except the cysteine 795 dropout. The fraction of strains with $|z|>2$ is shown for each amino acid dropout $(n=66)$. (E) The absence 796 of leucine or arginine leads to a large decrease in $C$. sporogenes relative abundance. Strains are colored 797 according to their rank-order abundance in the community grown in complete defined medium. Only strains 798 that were detected in at least one of the three samples were included $(n=92)$. $C$. sporogenes is highlighted 799 in black. Undetected strains were set to $10^{-7}$ for visualization. (F) C. sporogenes growth in complete defined 800 medium is dependent on the presence of arginine, and ornithine transcarbamoylase (otc) is partially 801 responsible for Arg metabolism. Wild type C. sporogenes and a $\Delta$ otc mutant were grown in complete 802 defined medium +/- Arg. Growth curves depict the mean of 3 replicates. Error bars represent 1 standard 803 deviation. (G) C. sporogenes requires otc to produce ATP from arginine. Intracellular ATP levels in C. 804 sporogenes incubated in PBS containing $2 \mathrm{mM}$ Arg are shown. (H) A proposed pathway for Arg metabolism 805 in C. sporogenes. Based on these data, we propose that Arg is converted to citrulline by the putative Arg 806 deiminase CLOSPO_00894; citrulline is then hydrolyzed to ornithine and carbamoyl phosphate by the 807 putative ornithine transcarbamoylase CLOSPO_02415, leading to the production of ATP. 


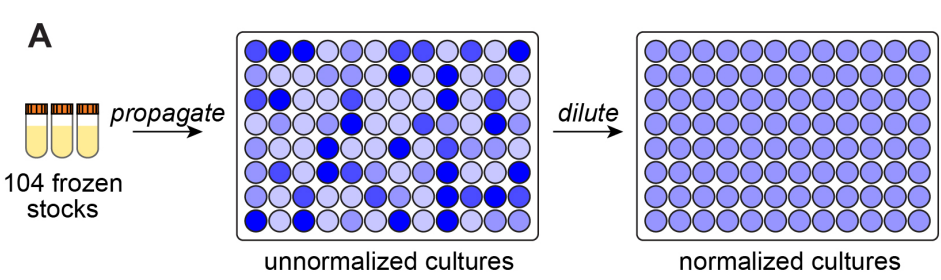

B

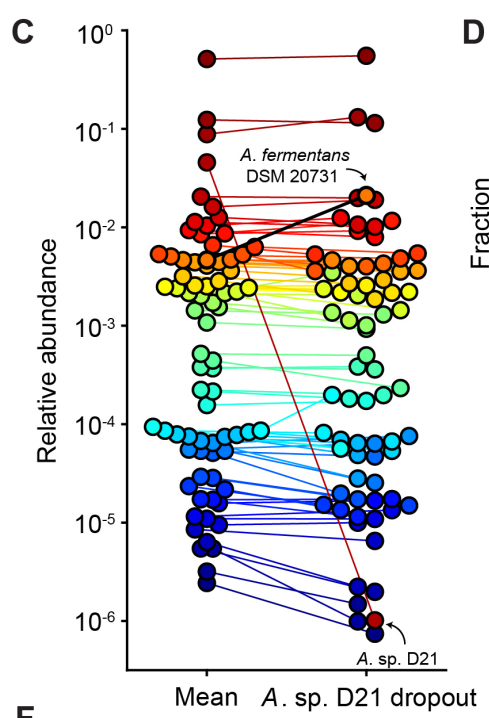

F

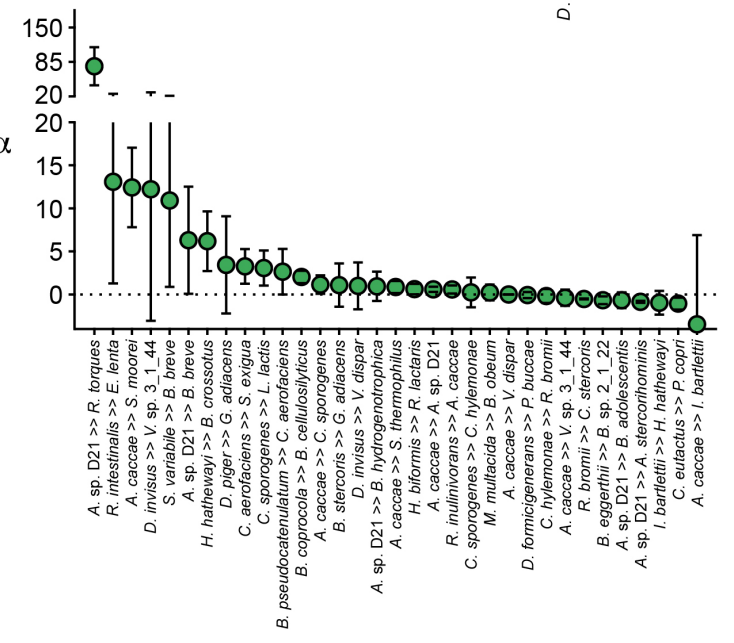

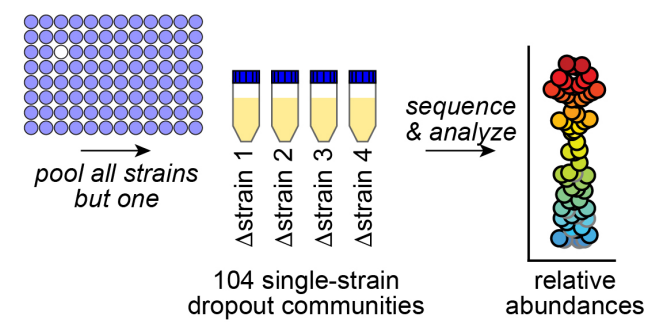
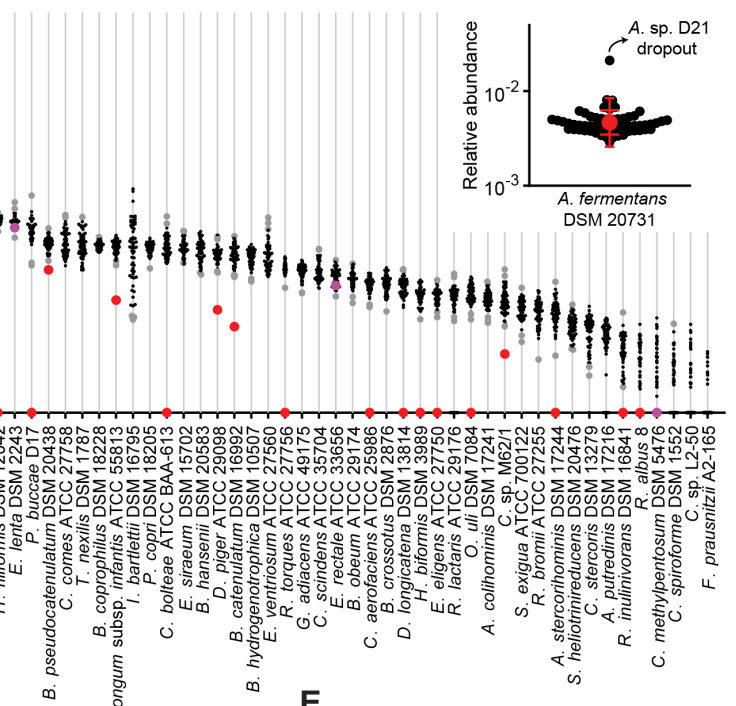

E

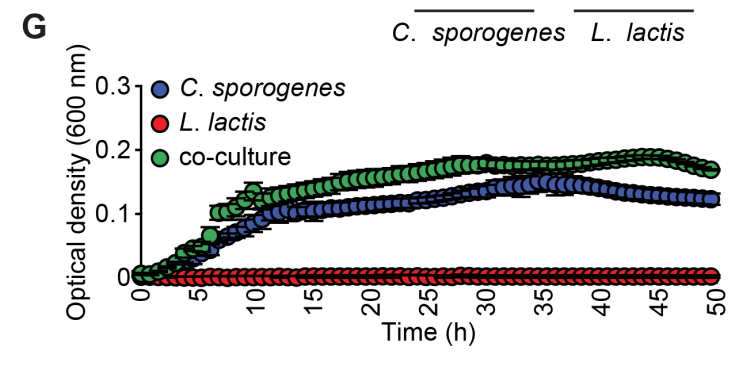
o. ind

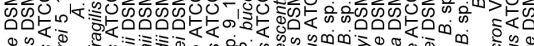

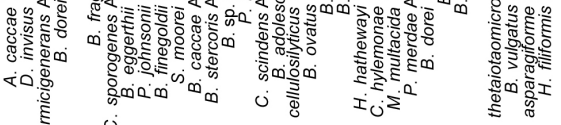
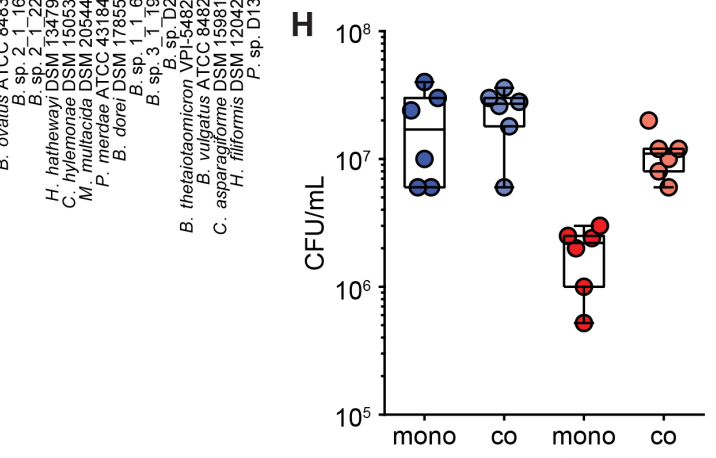
808 Figure 4: Systematic analysis of strain-strain interactions. (A) Schematic of the strain dropout 809 experiment. Frozen stocks of the strains were used to inoculate cultures that were grown for $24 \mathrm{~h}$, diluted 810 to similar optical densities (to the extent possible), and combined into 104 communities, each of which is 811 missing a single strain (i.e., 103-member communities). After $48 \mathrm{~h}$, communities were sequenced and 812 analyzed by NinjaMap to determine changes relative to the growth of the full 104-member community. (B) 813 Relative abundances for most strains are narrowly distributed. Each column depicts the relative abundance 814 of an individual strain across a set of 57 strain dropouts (black dots); the relative abundance of each strain 815 in its own dropout is shown as a red dot. Relative abundances in samples with $|z|>2$ are shown as gray 816 dots. Undetected strains were set to $10^{-7}$ for visualization. Inset: an enlarged view of the relative abundance 817 of Acidaminococcus fermentans DSM 20731 across 57 strain dropouts, showing that the elimination of 818 Acidaminococcus sp. D21 led to the expansion of $A$. fermentans DSM 20731. The red circle is the mean $819 \log _{10}$ (relative abundance); error bars show one (wide bar) or two (narrow bar) standard deviations from the 820 mean. (C) Response of the community to the removal of a single strain. Each dot is an individual strain; 821 the collection of dots in a column represents the community at a single timepoint. 76 strains are shown; 822 they are colored according to their rank-order mean $\log _{10}$ (relative abundance) across all samples in the 823 experimental group ( $n=57$ ). Both Acidaminococcus strains in the community are labeled; the removal of 824 Acidaminococcus sp. D21 leads to an increase in A. fermentans DSM 20731, with most other strains 825 staying at a similar level. (D) Removal of certain strains affected a large proportion of the community. The 826 effect of a strain dropout on each strain was determined by calculating the z-score across all samples 827 within an experimental group (Methods). The fraction of putative interactions was calculated based on the 828 strains with $|z|>2$ for each strain dropout ( $z<-2$ in blue, $z>2$ in red). Only strains above the limit of detection 829 in the experimental group were counted, thus $n$ is variable. (E) Some strains whose removal affected a 830 large portion of the community were at high relative abundance, others low. The plot shows the mean $831 \log _{10}$ (relative abundance) for each strain in the largest experimental group $(n=57)$, excluding the sample in 832 which that strain was dropped out. (F) A subset of the predicted interactions in the 104-member community 833 can be recapitulated in binary culture. Interaction scores were high for some strain pairs ( $a \rightarrow b$, where 834 removal of strain a affected strain $b$ ) with $z<-2.6$ (predicted positive interaction). We also included a pair 835 with slightly smaller $z$-score $(z=-2.1, C$. sporogenes $\rightarrow$ L. lactis). Data are the mean of 2-6 replicates, and 836 error bars represent 1 standard deviation from the mean. (G) C. sporogenes promotes L. lactis growth in 837 binary culture. Growth curves are plotted for mono- or co-cultures of C. sporogenes and L. lactis in 838 complete defined medium. Data are means and error bars represent 1 standard deviation from the mean $839(n=3)$. $(\mathbf{H})$ Colony forming units for both strains in mono- or co-culture in complete defined medium. $C$. 840 sporogenes levels were unaffected but the density of $L$. lactis increased $\sim 10$-fold in co-culture ( $p$-values 841 correspond to Student's t-tests $(n=6){ }^{* *}: p<0.05$. 


\section{REFERENCES}

843

844

845

846

847

848

849

850

851

852

853

854

855

856

857

858

859

860

861

862

863

864

865

866

867

868

869

870

871

872

873

874

875

876

877

878

879

880

881

882

883

884

885

886

887

888

889

890

891

892

893

894

Abreu, C.I., Friedman, J., Andersen Woltz, V.L., and Gore, J. (2019). Mortality causes universal changes in microbial community composition. Nat. Commun. 10, 2120.

Angly, F.E., Willner, D., Rohwer, F., Hugenholtz, P., and Tyson, G.W. (2012). Grinder: a versatile amplicon and shotgun sequence simulator. Nucleic Acids Res. 40, e94.

Aranda-Díaz, A., Ng, K.M., Thomsen, T., Real-Ramírez, I., Dahan, D., Dittmar, S., Gonzalez, C.G., Chavez, T., Vasquez, K.S., Nguyen, T.H., et al. (2020). High-throughput cultivation of stable, diverse, fecal-derived microbial communities to model the intestinal microbiota. BioRxiv.

Bai, Y., Müller, D.B., Srinivas, G., Garrido-Oter, R., Potthoff, E., Rott, M., Dombrowski, N., Münch, P.C., Spaepen, S., Remus-Emsermann, M., et al. (2015). Functional overlap of the Arabidopsis leaf and root microbiota. Nature 528, 364-369.

Bairey, E., Kelsic, E.D., and Kishony, R. (2016). High-order species interactions shape ecosystem diversity. Nat. Commun. 7, 12285.

Bankevich, A., Nurk, S., Antipov, D., Gurevich, A.A., Dvorkin, M., Kulikov, A.S., Lesin, V.M., Nikolenko, S.I., Pham, S., Prjibelski, A.D., et al. (2012). SPAdes: a new genome assembly algorithm and its applications to single-cell sequencing. J. Comput. Biol. 19, 455-477.

Blasche, S., Kim, Y., Oliveira, A.P., and Patil, K.R. (2017). Model microbial communities for ecosystems biology. Current Opinion in Systems Biology 6, 51-57.

Buffie, C.G., Bucci, V., Stein, R.R., McKenney, P.T., Ling, L., Gobourne, A., No, D., Liu, H., Kinnebrew, M., Viale, A., et al. (2015). Precision microbiome reconstitution restores bile acid mediated resistance to Clostridium difficile. Nature 517, 205-208.

Carlström, C.I., Field, C.M., Bortfeld-Miller, M., Müller, B., Sunagawa, S., and Vorholt, J.A. (2019). Synthetic microbiota reveal priority effects and keystone strains in the Arabidopsis phyllosphere. Nat. Ecol. Evol. 3, 1445-1454.

Chaumeil, P.-A., Mussig, A.J., Hugenholtz, P., and Parks, D.H. (2019). GTDB-Tk: a toolkit to classify genomes with the Genome Taxonomy Database. Bioinformatics.

Cullen, T.W., Schofield, W.B., Barry, N.A., Putnam, E.E., Rundell, E.A., Trent, M.S., Degnan, P.H., Booth, C.J., Yu, H., and Goodman, A.L. (2015). Antimicrobial peptide resistance mediates resilience of prominent gut commensals during inflammation. Science 347, 170-175.

Cunin, R., Glansdorff, N., Piérard, A., and Stalon, V. (1986). Biosynthesis and metabolism of arginine in bacteria. Microbiol Rev 50, 314-352.

Cuskin, F., Lowe, E.C., Temple, M.J., Zhu, Y., Cameron, E., Pudlo, N.A., Porter, N.T., Urs, K., Thompson, A.J., Cartmell, A., et al. (2015). Human gut Bacteroidetes can utilize yeast mannan through a selfish mechanism. Nature 517, 165-169.

Deschasaux, M., Bouter, K.E., Prodan, A., Levin, E., Groen, A.K., Herrema, H., Tremaroli, V., Bakker, G.J., Attaye, I., Pinto-Sietsma, S.-J., et al. (2018). Depicting the composition of gut microbiota in a population with varied ethnic origins but shared geography. Nat. Med. 24, 1526-1531.

Dethlefsen, L., and Relman, D.A. (2011). Incomplete recovery and individualized responses of the human distal gut microbiota to repeated antibiotic perturbation. Proc. Natl. Acad. Sci. USA 108 Suppl 1 , 


\section{4-4561.}

Dodd, D., Spitzer, M.H., Van Treuren, W., Merrill, B.D., Hryckowian, A.J., Higginbottom, S.K., Le, A., Cowan, T.M., Nolan, G.P., Fischbach, M.A., et al. (2017). A gut bacterial pathway metabolizes aromatic amino acids into nine circulating metabolites. Nature 551, 648-652.

Faith, J.J., McNulty, N.P., Rey, F.E., and Gordon, J.I. (2011). Predicting a human gut microbiota's response to diet in gnotobiotic mice. Science 333, 101-104.

Faith, J.J., Guruge, J.L., Charbonneau, M., Subramanian, S., Seedorf, H., Goodman, A.L., Clemente, J.C., Knight, R., Heath, A.C., Leibel, R.L., et al. (2013). The long-term stability of the human gut microbiota. Science $341,1237439$.

Franzosa, E.A., Huang, K., Meadow, J.F., Gevers, D., Lemon, K.P., Bohannan, B.J.M., and Huttenhower, C. (2015). Identifying personal microbiomes using metagenomic codes. Proc. Natl. Acad. Sci. USA 112, E2930-8.

Friedman, J., Higgins, L.M., and Gore, J. (2017). Community structure follows simple assembly rules in microbial microcosms. Nat. Ecol. Evol. 1, 109.

Funabashi, M., Grove, T.L., Wang, M., Varma, Y., McFadden, M.E., Brown, L.C., Guo, C., Higginbottom, S., Almo, S.C., and Fischbach, M.A. (2020). A metabolic pathway for bile acid dehydroxylation by the gut microbiome. Nature 582, 566-570.

Goldford, J.E., Lu, N., Bajić, D., Estrela, S., Tikhonov, M., Sanchez-Gorostiaga, A., Segrè, D., Mehta, P., and Sanchez, A. (2018). Emergent simplicity in microbial community assembly. Science 361, 469-474.

Goodman, A.L., McNulty, N.P., Zhao, Y., Leip, D., Mitra, R.D., Lozupone, C.A., Knight, R., and Gordon, J.I. (2009). Identifying genetic determinants needed to establish a human gut symbiont in its habitat. Cell Host Microbe 6, 279-289.

Goodman, A.L., Kallstrom, G., Faith, J.J., Reyes, A., Moore, A., Dantas, G., and Gordon, J.I. (2011). Extensive personal human gut microbiota culture collections characterized and manipulated in gnotobiotic mice. Proc. Natl. Acad. Sci. USA 108, 6252-6257.

Gopalakrishnan, V., Spencer, C.N., Nezi, L., Reuben, A., Andrews, M.C., Karpinets, T.V., Prieto, P.A., Vicente, D., Hoffman, K., Wei, S.C., et al. (2018). Gut microbiome modulates response to anti-PD-1 immunotherapy in melanoma patients. Science 359, 97-103.

Guo, C.-J., Allen, B.M., Hiam, K.J., Dodd, D., Van Treuren, W., Higginbottom, S., Nagashima, K., Fischer, C.R., Sonnenburg, J.L., Spitzer, M.H., et al. (2019). Depletion of microbiome-derived molecules in the host using Clostridium genetics. Science 366.

Gurevich, A., Saveliev, V., Vyahhi, N., and Tesler, G. (2013). QUAST: quality assessment tool for genome assemblies. Bioinformatics 29, 1072-1075.

Gutiérrez, N., and Garrido, D. (2019). Species Deletions from Microbiome Consortia Reveal Key Metabolic Interactions between Gut Microbes. MSystems 4.

Hart, S.F.M., Pineda, J.M.B., Chen, C.-C., Green, R., and Shou, W. (2019). Disentangling strictly selfserving mutations from win-win mutations in a mutualistic microbial community. Elife 8.

He, Y., Wu, W., Zheng, H.-M., Li, P., McDonald, D., Sheng, H.-F., Chen, M.-X., Chen, Z.-H., Ji, G.-Y., 
Zheng, Z.-D.-X., et al. (2018). Regional variation limits applications of healthy gut microbiome reference ranges and disease models. Nat. Med. 24, 1532-1535.

Hoek, T.A., Axelrod, K., Biancalani, T., Yurtsev, E.A., Liu, J., and Gore, J. (2016). Resource Availability Modulates the Cooperative and Competitive Nature of a Microbial Cross-Feeding Mutualism. PLoS Biol. $14, \mathrm{e} 1002540$.

Hsu, R.H., Clark, R.L., Tan, J.W., Romero, P.A., and Venturelli, O.S. (2019). Rapid microbial interaction network inference in microfluidic droplets: Supplementary Information. BioRxiv.

Kong, W., Meldgin, D.R., Collins, J.J., and Lu, T. (2018). Designing microbial consortia with defined social interactions. Nat. Chem. Biol. 14, 821-829.

Kraal, L., Abubucker, S., Kota, K., Fischbach, M.A., and Mitreva, M. (2014). The prevalence of species and strains in the human microbiome: a resource for experimental efforts. PLoS One 9, e97279.

Langmead, B., and Salzberg, S.L. (2012). Fast gapped-read alignment with Bowtie 2. Nat. Methods 9, 357-359.

Lebeis, S.L., Paredes, S.H., Lundberg, D.S., Breakfield, N., Gehring, J., McDonald, M., Malfatti, S., del Rio, T.G., Jones, C.D., Tringe, S.G., et al. (2015). Salicylic acid modulates colonization of the root microbiome by specific bacterial taxa. Science $349,860-864$.

Li, H., Handsaker, B., Wysoker, A., Fennell, T., Ruan, J., Homer, N., Marth, G., Abecasis, G., Durbin, R., and 1000 Genome Project Data Processing Subgroup (2009). The Sequence Alignment/Map format and SAMtools. Bioinformatics 25, 2078-2079.

Limoli, D.H., Warren, E.A., Yarrington, K.D., Donegan, N.P., Cheung, A.L., and O'Toole, G.A. (2019). Interspecies interactions induce exploratory motility in Pseudomonas aeruginosa. Elife 8.

Little, A.E.F., Robinson, C.J., Peterson, S.B., Raffa, K.F., and Handelsman, J. (2008). Rules of engagement: interspecies interactions that regulate microbial communities. Annu. Rev. Microbiol. 62, 375-401.

Lu, J., Breitwieser, F.P., Thielen, P., and Salzberg, S.L. (2017). Bracken: estimating species abundance in metagenomics data. PeerJ Computer Science 3, e104.

Martens, E.C., Kelly, A.G., Tauzin, A.S., and Brumer, H. (2014). The devil lies in the details: how variations in polysaccharide fine-structure impact the physiology and evolution of gut microbes. J. Mol. Biol. 426, 3851-3865.

Martínez, I., Maldonado-Gomez, M.X., Gomes-Neto, J.C., Kittana, H., Ding, H., Schmaltz, R., Joglekar, P., Cardona, R.J., Marsteller, N.L., Kembel, S.W., et al. (2018). Experimental evaluation of the importance of colonization history in early-life gut microbiota assembly. Elife 7.

Medlock, G.L., Carey, M.A., McDuffie, D.G., Mundy, M.B., Giallourou, N., Swann, J.R., Kolling, G.L., and Papin, J.A. (2018). Inferring Metabolic Mechanisms of Interaction within a Defined Gut Microbiota. Cell Syst. 7, 245-257.e7.

Mee, M.T., Collins, J.J., Church, G.M., and Wang, H.H. (2014). Syntrophic exchange in synthetic microbial communities. Proc. Natl. Acad. Sci. USA 111, E2149-56.

Morris, B.E.L., Henneberger, R., Huber, H., and Moissl-Eichinger, C. (2013). Microbial syntrophy: 
interaction for the common good. FEMS Microbiol. Rev. 37, 384-406.

Müller, B., and Grossniklaus, U. (2010). Model organisms--A historical perspective. J. Proteomics 73, 2054-2063.

Nayfach, S., Rodriguez-Mueller, B., Garud, N., and Pollard, K.S. (2016). An integrated metagenomics pipeline for strain profiling reveals novel patterns of bacterial transmission and biogeography. Genome Res. 26, 1612-1625.

Nayfach, S., Shi, Z.J., Seshadri, R., Pollard, K.S., and Kyrpides, N.C. (2019). New insights from uncultivated genomes of the global human gut microbiome. Nature 568, 505-510.

Ng, K.M., Aranda-Díaz, A., Tropini, C., Frankel, M.R., Van Treuren, W., O'Loughlin, C.T., Merrill, B.D., Yu, F.B., Pruss, K.M., Oliveira, R.A., et al. (2019). Recovery of the Gut Microbiota after Antibiotics Depends on Host Diet, Community Context, and Environmental Reservoirs. Cell Host Microbe 26, 650665.e4.

Nisman, B. (1954). The Stickland reaction. Bacteriol Rev 18, 16-42.

O'Leary, N.A., Wright, M.W., Brister, J.R., Ciufo, S., Haddad, D., McVeigh, R., Rajput, B., Robbertse, B., Smith-White, B., Ako-Adjei, D., et al. (2016). Reference sequence (RefSeq) database at NCBI: current status, taxonomic expansion, and functional annotation. Nucleic Acids Res. 44, D733-45.

Pacheco, A.R., and Segrè, D. (2019). A multidimensional perspective on microbial interactions. FEMS Microbiol. Lett. 366.

Parks, D.H., Imelfort, M., Skennerton, C.T., Hugenholtz, P., and Tyson, G.W. (2015). CheckM: assessing the quality of microbial genomes recovered from isolates, single cells, and metagenomes. Genome Res. 25, 1043-1055.

Parks, D.H., Chuvochina, M., Waite, D.W., Rinke, C., Skarshewski, A., Chaumeil, P.-A., and Hugenholtz, P. (2018). A standardized bacterial taxonomy based on genome phylogeny substantially revises the tree of life. Nat. Biotechnol. 36, 996-1004.

Parks, D.H., Chuvochina, M., Chaumeil, P.-A., Rinke, C., Mussig, A.J., and Hugenholtz, P. (2020). A complete domain-to-species taxonomy for Bacteria and Archaea. Nat. Biotechnol. 38, 1079-1086.

Patnode, M.L., Beller, Z.W., Han, N.D., Cheng, J., Peters, S.L., Terrapon, N., Henrissat, B., Le Gall, S., Saulnier, L., Hayashi, D.K., et al. (2019). Interspecies Competition Impacts Targeted Manipulation of Human Gut Bacteria by Fiber-Derived Glycans. Cell 179, 59-73.e13.

Qin, J., Li, R., Raes, J., Arumugam, M., Burgdorf, K.S., Manichanh, C., Nielsen, T., Pons, N., Levenez, F., Yamada, T., et al. (2010). A human gut microbial gene catalogue established by metagenomic sequencing. Nature 464, 59-65.

Qin, M., Wu, S., Li, A., Zhao, F., Feng, H., Ding, L., Chang, Y., and Ruan, J. (2018). Lrscaf: improving draft genomes using long noisy reads. BioRxiv.

Ridaura, V.K., Faith, J.J., Rey, F.E., Cheng, J., Duncan, A.E., Kau, A.L., Griffin, N.W., Lombard, V., Henrissat, B., Bain, J.R., et al. (2013). Gut microbiota from twins discordant for obesity modulate metabolism in mice. Science 341, 1241214.

Routy, B., Le Chatelier, E., Derosa, L., Duong, C.P.M., Alou, M.T., Daillère, R., Fluckiger, A., 
054

055

056

057

058

059

060

061

062

063

064

065

066

067

068

069

070

071

072

073

074

075

076

077

078

079

080

081

082

083

084

085

086

087

088

089

090

091

092

093

094

095

096

097

098

099

100

101

102

103

104

105

106

Messaoudene, M., Rauber, C., Roberti, M.P., et al. (2018). Gut microbiome influences efficacy of PD-1based immunotherapy against epithelial tumors. Science 359, 91-97.

Sanchez-Gorostiaga, A., Bajić, D., Osborne, M.L., Poyatos, J.F., and Sanchez, A. (2019). High-order interactions distort the functional landscape of microbial consortia. PLoS Biol. 17, e3000550.

Shank, E.A., and Kolter, R. (2009). New developments in microbial interspecies signaling. Curr. Opin. Microbiol. 12, 205-214.

Shen, W., Le, S., Li, Y., and Hu, F. (2016). SeqKit: A Cross-Platform and Ultrafast Toolkit for FASTA/Q File Manipulation. PLoS One 11, e0163962.

Sinha, R., Stanley, G., Gulati, G.S., Ezran, C., Travaglini, K.J., Wei, E., Chan, C.K.F., Nabhan, A.N., Su, T., Morganti, R.M., et al. (2017). Index switching causes "spreading-of-signal" among multiplexed samples in Illumina HiSeq 4000 DNA sequencing. BioRxiv.

Smith, E.A., and Macfarlane, G.T. (1997). Dissimilatory amino Acid metabolism in human colonic bacteria. Anaerobe 3, 327-337.

Sonnenburg, E.D., and Sonnenburg, J.L. (2019). The ancestral and industrialized gut microbiota and implications for human health. Nat. Rev. Microbiol. 17, 383-390.

Sonnenburg, E.D., Zheng, H., Joglekar, P., Higginbottom, S.K., Firbank, S.J., Bolam, D.N., and Sonnenburg, J.L. (2010). Specificity of polysaccharide use in intestinal bacteroides species determines diet-induced microbiota alterations. Cell 141, 1241-1252.

Soto-Martin, E.C., Warnke, I., Farquharson, F.M., Christodoulou, M., Horgan, G., Derrien, M., Faurie, J.M., Flint, H.J., Duncan, S.H., and Louis, P. (2020). Vitamin Biosynthesis by Human Gut ButyrateProducing Bacteria and Cross-Feeding in Synthetic Microbial Communities. MBio 11.

Titus Brown, C., and Irber, L. (2016). sourmash: a library for MinHash sketching of DNA. JOSS 1.

Traxler, M.F., Watrous, J.D., Alexandrov, T., Dorrestein, P.C., and Kolter, R. (2013). Interspecies interactions stimulate diversification of the Streptomyces coelicolor secreted metabolome. MBio 4.

Truong, D.T., Franzosa, E.A., Tickle, T.L., Scholz, M., Weingart, G., Pasolli, E., Tett, A., Huttenhower, C., and Segata, N. (2015). MetaPhIAn2 for enhanced metagenomic taxonomic profiling. Nat. Methods 12, 902-903.

Venugopal, V., and Nadkarni, G.B. (1977). Regulation of the arginine dihydrolase pathway in Clostridium sporogenes. J. Bacteriol. 131, 693-695.

Vetsigian, K., Jajoo, R., and Kishony, R. (2011). Structure and evolution of Streptomyces interaction networks in soil and in silico. PLoS Biol. 9, e1001184.

Walter, J., Maldonado-Gómez, M.X., and Martínez, I. (2018). To engraft or not to engraft: an ecological framework for gut microbiome modulation with live microbes. Curr. Opin. Biotechnol. 49, 129-139.

Wexler, A.G., and Goodman, A.L. (2017). An insider's perspective: Bacteroides as a window into the microbiome. Nat. Microbiol. 2, 17026.

Wick, R.R., Judd, L.M., Gorrie, C.L., and Holt, K.E. (2017). Unicycler: Resolving bacterial genome assemblies from short and long sequencing reads. PLoS Comput. Biol. 13, e1005595. 
Widder, S., Allen, R.J., Pfeiffer, T., Curtis, T.P., Wiuf, C., Sloan, W.T., Cordero, O.X., Brown, S.P., Momeni, B., Shou, W., et al. (2016). Challenges in microbial ecology: building predictive understanding of community function and dynamics. ISME J. 10, 2557-2568.

Wildenauer, F.X., and Winter, J. (1986). Fermentation of isoleucine and arginine by pure and syntrophic cultures of Clostridium sporogenes. FEMS Microbiol. Lett. 38, 373-379.

Wood, D.E., Lu, J., and Langmead, B. (2019). Improved metagenomic analysis with Kraken 2. Genome Biol. 20, 257.

Xavier, J.B. (2011). Social interaction in synthetic and natural microbial communities. Mol. Syst. Biol. 7, 483.

Xu, M., Guo, L., Gu, S., Wang, O., Zhang, R., Fan, G., Xu, X., Deng, L., and Liu, X. (2019). TGSGapCloser: fast and accurately passing through the Bermuda in large genome using error-prone thirdgeneration long reads. BioRxiv.

Yurtsev, E.A., Conwill, A., and Gore, J. (2016). Oscillatory dynamics in a bacterial cross-protection mutualism. Proc. Natl. Acad. Sci. USA 113, 6236-6241.

Ze, X., Duncan, S.H., Louis, P., and Flint, H.J. (2012). Ruminococcus bromii is a keystone species for the degradation of resistant starch in the human colon. ISME J. 6, 1535-1543.

Ziesack, M., Gibson, T., Oliver, J.K.W., Shumaker, A.M., Hsu, B.B., Riglar, D.T., Giessen, T.W., DiBenedetto, N.V., Bry, L., Way, J.C., et al. (2019). Engineered Interspecies Amino Acid Cross-Feeding Increases Population Evenness in a Synthetic Bacterial Consortium. MSystems 4.

Zipperer, A., Konnerth, M.C., Laux, C., Berscheid, A., Janek, D., Weidenmaier, C., Burian, M., Schilling, N.A., Slavetinsky, C., Marschal, M., et al. (2016). Human commensals producing a novel antibiotic impair pathogen colonization. Nature 535, 511-516. 